\title{
FACTORIZATION RINGS
}

\author{
J.-M. MARANDA
}

1. Introduction. Let $\mathfrak{o}$ be an integral domain with $\Omega$ as field of quotients. W. Krull has shown $(3 ; 4)$ that the following three conditions on $\mathfrak{v}$ are equivalent:

1. There is a set of rank 1 , discrete valuations of $\Re,\left\{V_{i}\right\}_{i \in I}$, such that for each non-null element $a \in \Omega, V_{i}(a)=0$ for all $i \in I$ except a finite number, and such that for all $a \in \Re, a \in \mathbb{D}$ if and only if $V_{i}(a) \geqslant 0$ for all $i \in I$.

2. Every non-trivial principal ideal of $\mathfrak{D}$ is the intersection of a finite number of formal powers of minimal non-trivial prime ideals of $\mathfrak{o}$.

3. The partially ordered semi-group of classes of quasi-equal non-null ideals (fractional) of $\mathfrak{D}$ is a group with unique factorization theorem.

Krull called an integral domain $\mathbf{o}$ satisfying these conditions an "endliche diskrete Hauptordnung" and showed that there is a minimum set of rank 1, discrete valuations of $\Omega$ satisfying 1 . We may notice that a Dedekind ring is an "endliche diskrete Hauptordnung" for which the theory of quasi-equality is trivial, i.e. if two ideals are quasi-equal, then they are equal.

The object of this paper is to generalize this theory of integral domains to a theory of arbitrary commutative rings with unity element. For simplicity, we will call these generalized "endliche diskrete Hauptordnungen" "factorization rings"'.

The reader will soon realize that the theory of quasi-equality of van der Waerden and Artin $(7, \S 105)$, generalized to the case of an arbitrary commutative ring with unity element, is the fundamental tool utilized.

We will obtain in particular, those known results concerning Noetherian rings that are integrally closed in their full ring of quotients, that are given in $(5, \$ 4.7$ and $\$ 4.9)$, most of them in a more general context (the ascending chain condition is not necessarily valid for the ideals of a factorization ring), and by methods that are undoubtedly "multiplicative."

In $\$ 6$ we will define the notion of a "generalized Dedekind ring", and although we cannot go into any details here in the introduction, we may remark that for such a generalized Dedekind ring $\mathfrak{b}$, if the ascending chain condition is valid for its ideals, then, for any ideal $\mathfrak{a}$ of $\mathfrak{b}$,

$$
\mathfrak{a}=\mathfrak{a}_{S} \mathfrak{p}_{1}^{m_{1}} \mathfrak{p}_{2}^{m_{2}} \ldots \mathfrak{p}_{r}^{m_{r}}
$$

where $S$ is the set of all regular elements of $\mathfrak{o}$, where $\mathfrak{a}_{S}$ is the isolated component of $\mathfrak{a}$ determined by $S$ and where the $\mathfrak{p}_{i}$ are relevant prime ideals (5, p. 76) of $\mathrm{b}$, and this decomposition is "unique" in a certain sense. This is an

Received August 15, 1956; in revised form October 2, 1956. 
obvious generalization of the unique decomposition theorem for the ideals of an ordinary Dedekind ring.

Finally, if $\mathfrak{D}$ is a commutative ring with unity element, and if the descending chain condition is valid for the ideals of $\mathfrak{D}$, then we will determine, in $\S 7$, all orders of $\mathfrak{D}$ that are Noetherian generalized Dedekind rings.

2. Valuations and Subvaluations. Let us consider a commutative and associative ring $\mathfrak{O}$.

Definition. A function $V$ of $\mathfrak{D}$ onto a partially ordered semi-group $M$ will be called a valuation ${ }^{1}$ of $\mathfrak{O}$ if for all $a, b, c \in \mathfrak{O}$,

V1.

V2.

$$
\begin{aligned}
& V(a) \leqslant V(b) \& V(a) \leqslant V(c) \rightarrow V(a) \leqslant V(b-c) \\
& V(a b)=V(a) V(b)
\end{aligned}
$$

We will call $M$ the ordered semi-group of values of $V$. In the case where $M$ is totally ordered, V1 may be replaced by

V1'.

$$
V(b-c) \geqslant \min \{V(b), V(c)\}
$$

Definition. A reflexive and transitive binary relation $R$ on $\mathfrak{D}$ will be called a subvaluation of $\mathfrak{D}$ if for all $a, b, c, d \in \mathfrak{D}$,

S1.

$a R b \& a R c \rightarrow a R(b-c)$

S2.

$a R b \& c R d \rightarrow a c R b d$

If $V$ is a valuation of $\mathfrak{D}$ and if we define the relation $R$ on $\mathfrak{D}$ as follows: for all $a, b \in \mathfrak{O}, a R b$ if and only if $V(a) \leqslant V(b)$, then it is easily verified that $R$ is a subvaluation of $\mathfrak{O}$. We will say that $R$ is the subvaluation of $\mathfrak{D}$ determined by $V$.

Conversely, let $R$ be a subvaluation of $\mathfrak{O}$. If we define the relation $\widetilde{R}$ on $\mathfrak{O}$ as follows: for all $a, b \in \mathfrak{O}, a \widetilde{R} b$ if and only if $a R b$ and $b R a$, then one can verify the following:

1. The relation $\widetilde{R}$ is an equivalence relation and if $V$ is the natural function of $\mathfrak{O}$ onto the quotient set $M=\mathfrak{D} / \widetilde{R}$, then one may define a partial ordering relation on $M$ as follows: for all $a, b \in \mathfrak{O}, V(a) \leqslant V(b)$ if and only if $a R b$.

2 . The relation $\widetilde{R}$ is multiplicative so that one can define an induced multiplication on $M$, and with respect to this operation and the partial ordering relation defined above, $M$ is a partially ordered semi-group.

3 . The function $V$ is a valuation of $\subseteq$ with $M$ as ordered semi-group of values and $V$ determines the given subvaluation $R$ of $\mathfrak{D}$.

Let $V$ be a valuation of $\mathfrak{O}$ with ordered semi-group of values $M$ and let $R$ be the subvaluation of $\mathfrak{O}$ determined by $V$. For all $a \in \mathfrak{O}$, we have $a R 0$ and $a R(-a)$, for, since $R$ is reflexive, $a R a$, and by $\mathrm{S} 1$,

$$
\begin{aligned}
& a R a \& a R a \rightarrow a R(a-a) \\
& a R 0 \& a R a \rightarrow a R(0-a)
\end{aligned}
$$

\footnotetext{
In the case where $\mathfrak{D}$ is a field and $M$ is a partially ordered group with added symbol $\infty$, this definition is not new, see e.g. (2).
} 
The corresponding properties for $V$ are: for all $a \in \mathfrak{D}, V(a) \leqslant V(0)$ and $V(a) \leqslant V(-a)$ so that $V(a)=V(-a)$.

Proposition 1. If

$$
\mathfrak{a}=\{a \in \mathfrak{D} \mid 0 R a\}=\{a \in \mathfrak{D} \mid V(a)=V(0)\}
$$

then $\mathfrak{a}$ is an ideal of $\mathfrak{D}$ which we will call the kernel of $V$. If $a, b \in \mathfrak{D}$, then

$$
a \equiv b(\bmod \mathfrak{a}) \rightarrow V(a)=V(b)
$$

Proof. If $a, b \in \mathfrak{a}$, then $0 R a$ and $0 R b$ so that by $\mathrm{S} 1,0 R(a-b)$ and $a-b \in \mathfrak{a}$. If $a \in \mathfrak{a}$ and $b \in \mathfrak{D}$, then $0 R a$ and $b R b$ so that by $\mathrm{S} 2,0 b R a b$ therefore, $a b \in \mathfrak{a}$.

Now if $a, b \in \mathfrak{D}$ and if $a-b \in \mathfrak{a}$, then $0 R(a-b)$, and since $R$ is transitive,

$$
\begin{aligned}
& a R 0 \& 0 R(a-b) \rightarrow a R(a-b) \\
& b R 0 \& 0 R(a-b) \rightarrow b R(a-b)
\end{aligned}
$$

Then, by S1,

$$
\begin{aligned}
& a R a \& a R(a-b) \rightarrow a R(a-(a-b)) \\
& b R(a-b) \& b R(-b) \rightarrow b R((a-b)-(-b))
\end{aligned}
$$

i.e. $a R b$ and $b R a$ so that $V(a) \leqslant V(b)$ and $V(b) \leqslant V(a)$ and therefore, $V(a)=V(b)$.

We may notice that if $a, b \in \mathfrak{D}$ and if $V(a)=V(b)$, it is not necessarily true that $a$ is congruent to $b$, modulo $a$.

Now if $\phi$ is a homomorphism of $\mathfrak{D}$ onto a ring $\mathfrak{D}^{\prime}$, and if the kernel of $\phi$ is contained in the kernel $a$ of $V$, then, by Proposition 1, one can define a function $V^{\prime}$ of $\mathfrak{D}^{\prime}$ onto $M$ by setting $V^{\prime}(\phi(a))=V(a)$ for all $a \in \mathfrak{D}$, and it can easily be verified that $V^{\prime}$ is a valuation of $\mathfrak{D}^{\prime}$ with $M$ as ordered semi-group of values. We will call $V^{\prime}$ the projection of $V$ by $\phi$. Notice that the kernel of $V^{\prime}$ is just $\phi(\mathfrak{a})$.

Conversely, if $V^{\prime}$ is a valuation of $\mathfrak{O}^{\prime}$ and if for all $a \in \mathfrak{D}$ one sets $V(a)=$ $V^{\prime}(\phi(a))$, one can easily verify that $V$ is a valuation of $\mathfrak{D}$ and that the kernel of $\phi$ is contained in the kernel of $V$.

If $\mathfrak{D}$ is a commutative ring with unity element, then the relation of divisibility " $a$ divides $b$ if and only if there exists an element $c \in \mathfrak{D}$ such that $b=a c$ " is evidently a subvaluation of $\mathfrak{D}$. By this definition of divisibility, every element of $\mathfrak{D}$ divides 0 so that we will use the term "regular element" to denote those elements $a \in \mathfrak{D}$ that have the properties " $a \neq 0$ and for all $b \in \mathfrak{O}, a b=0$ implies that $b=0$," instead of the usual term "non-divisor of zero."

From now on, $\mathfrak{D}$ will always denote a commutative ring with unity element in which every regular element is invertible. Also, $G$ will denote the totally ordered additive group of ordinary integers, $G^{\prime}$ will denote the totally ordered semi-group obtained by adding the symbol $\infty$ to $G$ with the laws

1. for all $u \in G^{\prime}, u \leqslant \infty$;

2. for all $u \in G^{\prime}, u+\infty=\infty+u=\infty$; 
and $G^{\prime \prime}$ will denote the totally ordered semi-group obtained by adding the symbol $\infty^{\prime}$ to $G^{\prime}$ with the laws:

1. for all $u \in G^{\prime \prime}, \infty^{\prime} \leqslant u$;

2. for all $u \in G, \infty^{\prime}+u=u+\infty^{\prime}=\infty^{\prime}$;

3. $\infty^{\prime}+\infty^{\prime}=\infty^{\prime}$;

4. $\infty^{\prime}+\infty=\infty+\infty^{\prime}=\infty$.

DeFinition. We will say that a valuation $V$ of $\leqq$ is special if $G^{\prime}$ is the ordered semi-group of values of $V$ and if there exists a regular element a $\in \mathfrak{O}$ such that $V(a)>0$.

If $V$ is a special valuation of $\mathfrak{D}$, there exists an element $a \in \mathfrak{D}$ such that $V(a) \neq \infty$ and then,

$$
V(a)=V(1 a)=V(1)+V(a)
$$

so that $V(1)=0$. Also, if $a$ is a regular element of $\mathfrak{D}$, then

$$
0=V(1)=V\left(a a^{-1}\right)=V(a)+V\left(\bar{a}^{-1}\right),
$$

so that $V(a) \neq \infty$.

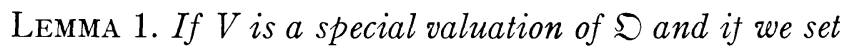

$$
\mathfrak{D}=\{a \in \mathfrak{D} \mid V(a) \geqslant 0\}
$$

then $\mathfrak{D}$ is an order of $\mathfrak{D}$. We will say that $\mathfrak{D}$ is the order of $\mathfrak{O}$ determined by $V$.

If $\mathfrak{D}^{\prime}$ is any order of $\mathfrak{D}$ with the property that for all $a \in \mathfrak{D}^{\prime}, V(a) \geqslant 0$, and if for each positive integer $n$ we set

$$
\mathfrak{q}_{n}=\left\{a \in \mathfrak{o}^{\prime} \mid V(a) \geqslant n\right\}
$$

then $\mathfrak{p}=\mathfrak{q}_{1}$ is a proper prime ideal of $\mathfrak{D}^{\prime}$ containing a regular element and the $\mathfrak{q}_{n}$ are all $\mathfrak{p}$-primary ideals of $\mathfrak{b}^{\prime}$. Furthermore, if we set

$$
\mathfrak{p}^{\prime}=\left\{a \in \mathfrak{v}^{\prime} \mid V(a)=\infty\right\}
$$

then $\mathfrak{p}^{\prime}$ is a prime ideal of $\mathfrak{b}^{\prime}$ and

$$
\mathfrak{p}^{\prime}=\bigcap_{n=1}^{\infty} \mathfrak{q}_{n}
$$

Finally, if $\mathfrak{D}=\mathfrak{v}^{\prime}$, the $\mathfrak{q}_{n}$ are all distinct and $\mathfrak{p}^{\prime}$ is a prime ideal of $\mathfrak{D}$.

Proof. If $a, b \in \mathfrak{D}$, then $V(a) \geqslant 0$ and $V(b) \geqslant 0$ so that

$$
V(a-b) \geqslant \min \{V(a), V(b)\} \geqslant 0, V(a b)=V(a)+V(b) \geqslant 0
$$

and therefore, $a-b, a b \in \mathfrak{D}$. Since $V(1)=0,1 \in \mathfrak{D}$. By the definition of a special valuation, there exists a regular element $a \in \mathfrak{S}$ such that $V(a)>0$ so that $a \in \mathfrak{D}$ and if $b$ is any element of $\mathfrak{v}$ that is not in $\mathfrak{v}$, then there exists a positive integer $n$ such that $V\left(a^{n}\right)=n V(a) \geqslant-V(b)$ so that

$$
V\left(a^{n} b\right)=V\left(a^{n}\right)+V(b) \geqslant 0
$$

and $a^{n} b \in \mathfrak{D}$. We have thus shown that $\mathfrak{D}$ is an order of $\mathfrak{D}$. 
If $a, b \in \mathfrak{q}_{n}$, then $V(a) \geqslant n$ and $V(b) \geqslant n$ and therefore,

$$
V(a-b) \geqslant \min \{V(a), V(b)\} \geqslant n
$$

so that $a-b \in \mathfrak{q}_{n}$. If $a \in \mathfrak{q}_{n}$ and $b \in \mathfrak{o}^{\prime}$, then $V(a) \geqslant n$ and $V(b) \geqslant 0$ so that

$$
V(a b)=V(a)+V(b) \geqslant n
$$

and therefore, $a b \in \mathfrak{q}_{n}$. The $\mathfrak{q}_{n}$ are thus ideals of $\mathfrak{v}^{\prime}$.

If $a \in \mathfrak{p}$, then $V(a) \geqslant 1$, so that $V\left(a^{n}\right)=n V(a) \geqslant n$ and therefore, $a^{n} \in \mathfrak{q}_{n}$. Therefore, $\mathfrak{p} \subseteq \operatorname{rad} \mathfrak{q}_{n}$. If $a, b \in \mathfrak{b}^{\prime}, a \notin \mathfrak{p}$ and $a b \in \mathfrak{q}_{n}$, then $V(a)=0$ and $V(a b) \geqslant n$ so that

$$
V(b)=V(a)+V(b)=V(a b) \geqslant n
$$

and therefore, $b \in \mathfrak{q}_{n}$. This proves that $\mathfrak{p}$ is a prime ideal of $\mathfrak{o}^{\prime}$ and that each $\mathfrak{q}_{n}$ is $\mathfrak{p}$-primary. Since $V(1)=0,1 \notin \mathfrak{p}$ so that $\mathfrak{p}$ is a proper ideal of $\mathfrak{p}^{\prime}$. By the definition of a special valuation, there exists a regular element $a \in \mathbb{D}$ such that $V(a)>0$, and since $\mathfrak{D}^{\prime}$ is an order of $\mathfrak{D}$, there exists a regular element $b \in \mathfrak{D}^{\prime}$ such that $b a=c \in \mathfrak{o}^{\prime}$. Then $c$ is a regular element of $\mathfrak{o}^{\prime}$ and $V(c)=$ $V(b)+V(a)>0$ so that $c \in p$.

It is evident that

$$
\mathfrak{p}^{\prime}=\bigcap_{n=1}^{\infty} \mathfrak{q}_{n}
$$

so that $\mathfrak{p}^{\prime}$ is an ideal of $\mathfrak{v}^{\prime}$. If $a$ and $b$ are elements of $\mathfrak{v}^{\prime}$ that are not in $\mathfrak{p}^{\prime}$, then $V(a) \neq \infty \neq V(b)$ so that $V(a b)=V(a)+V(b) \neq \infty$ and therefore, $a b \notin \mathfrak{p}^{\prime}$. Thus, $\mathfrak{p}^{\prime}$ is a prime ideal of $\mathfrak{b}^{\prime}$.

If $\mathfrak{v}=\mathfrak{o}^{\prime}$, then evidently,

$$
\mathfrak{p}^{\prime}=\{a \in \mathfrak{D} \mid V(a)=\infty\} .
$$

This means that $\mathfrak{p}^{\prime}$ is the kernel of $V$ so that it is an ideal of $\mathfrak{D}$. Just as above, one may then show that $\mathfrak{p}^{\prime}$ is a prime ideal of $\mathfrak{D}$. Finally, if $n$ is any positive integer, there exists an element $a \in \mathfrak{D}$ such that $V(a)=n$. This means that $a \in \mathfrak{q}_{n}$ and $a \notin \mathfrak{q}_{n+1}$. Therefore, the $\mathfrak{q}_{n}$ are all distinct.

3. The Theory of Quasi-Divisibility. In this section, we give an account of the theory of quasi-divisibility of van der Waerden and Artin, generalized to the case of an arbitrary commutative ring with unity element. The proofs are easy generalizations of those given in $(7, \S 105)$ and will be left to the reader.

Let $\mathfrak{D}$ be an order of $\mathfrak{D}$. By an $\mathfrak{D}$-ideal of $\mathfrak{D}$, we mean simply an $\mathfrak{D}$-submodule of $\mathfrak{D}$, and we denote the set of all $\mathfrak{D}$-ideals of $\mathfrak{D}$ by $\mathfrak{L}(\mathfrak{D})$. The ordinary ideals of $\mathfrak{D}$ are just those $\mathfrak{D}$-ideals of $\mathfrak{D}$ that are contained in $\mathfrak{D}$; we will call these the integral $\mathfrak{D}$-ideals of $\mathfrak{D}$ or just simply the ideals of $\mathfrak{D}$.

If as usual, one defines the product of two $\mathfrak{D}$-ideals $\mathfrak{a}$ and $\mathfrak{b}$ of $\mathfrak{D}$ to be the D-ideal of $\mathfrak{D}$ generated by the set of all products $a b$, where $a \in \mathfrak{a}$ and $b \in \mathfrak{b}$, 
then with respect to this operation and ordinary set inclusion, $\mathfrak{R}(\mathfrak{D})$ is a commutative partially ordered semi-group with the following properties:

1. $\mathfrak{D}$ is the unity element of $\mathfrak{R}(\mathfrak{D})$;

2. The null ideal $(0)$ is the zero element of $\mathfrak{R}(\mathfrak{D})$;

3. $\mathfrak{R}(\mathfrak{D})$ is complete and distributive, i.e., if $\left\{\mathfrak{a}_{i}\right\}_{i \in I}$ is a set of elements of $\mathfrak{R}(\mathfrak{D})$, then $\Sigma \mathfrak{a}_{i}$ (the sum of the $\mathfrak{a}_{i}$ considered as $\mathfrak{D}$-submodules of $\mathfrak{D}$ ) is the least upper bound of $\left\{\mathfrak{a}_{i}\right\}_{i \in I}$;

$$
\bigcap_{i \in I} \mathfrak{a}_{i}
$$

(set-theoretical intersection) is the greatest lower bound of $\left\{a_{i}\right\}_{i \in I}$ and if $\mathfrak{a} \in \mathfrak{R}(\mathfrak{D})$, then

$$
\mathfrak{a} \sum_{i \in I} \mathfrak{a}_{i}=\sum_{i \in I} \mathfrak{a}_{i} .
$$

If $\mathfrak{a} \in \mathfrak{R}(\mathfrak{D})$, we denote by $\mathfrak{a}^{-1}$ the set of all $a \in \mathfrak{D}$ such that $a \mathfrak{a} \subseteq \mathfrak{v}$. One may show that $\mathfrak{a}^{-1}$ is the largest $\mathfrak{D}$-ideal $\mathfrak{b}$ of $\mathfrak{D}$ with the property $\mathfrak{b a} \subseteq \mathfrak{D}$, and if $\mathfrak{a}$ is invertible, then its inverse is $\mathfrak{a}^{-1}$. If $a$ is a regular element of $\mathfrak{b}$, then $(a)^{-1}=\left(a^{-1}\right)$.

Definition. If $\mathfrak{a}, \mathfrak{b} \in \mathfrak{R}(\mathfrak{b})$, we say that $\mathfrak{a}$ quasi-divides $\mathfrak{b}$ and write $\mathfrak{a} \leqslant \mathfrak{b}$, if $\mathfrak{a}^{-1} \subseteq \mathfrak{b}^{-1}$.

Let us notice that $\mathfrak{a} \subseteq \mathfrak{b}$ implies that $\mathfrak{a} \geqslant \mathfrak{b}$.

The relation of quasi-divisibility is a reflexive and transitive relation on $\mathcal{R}(\mathfrak{D})$, and furthermore, it is multiplicative. We may then define a relation of "quasi-equality" on $\mathfrak{R}(\mathfrak{b})$ as follows: $\mathfrak{a}$ is quasi-equal to $\mathfrak{b}$, which we write $\mathfrak{a} \sim \mathfrak{b}$, if $\mathfrak{a} \leqslant \mathfrak{b}$ and $\mathfrak{b} \leqslant \mathfrak{a}$, i.e. if $\mathfrak{a}^{-1}=\mathfrak{b}^{-1}$, and this relation is a multiplicative equivalence relation on $\mathfrak{R}(\mathfrak{D})$. We will denote with $\overline{\mathfrak{R}(\mathfrak{D})}$ the set of equivalence classes of $\mathfrak{R}(\mathfrak{D})$, determined by the relation of quasi-equality, and if $\mathfrak{a} \in \mathfrak{R}(\mathfrak{D})$, we will denote by $\overline{\mathfrak{a}}$ that class in $\overline{\mathfrak{Q}(\mathfrak{D})}$ that contains $\mathfrak{a}$. Since the relation of quasi-equality is multiplicative, one can define an induced product on $\overline{Q(\mathfrak{D})}$ and the relation of quasi-divisibility induces a partial ordering relation on $\overline{\mathbb{Q}(0)}$, which we denote with the same symbol " $\leqslant$ ", and which is multiplicative, so that $\overline{\mathbb{R ( D )}}$ is a partially ordered semi-group with $\overline{\mathfrak{D}}$ as unity element and $\overline{(0)}$ as zero element.

Proposition 2. If $\mathfrak{a}, \mathfrak{b} \in \mathfrak{R}(\mathfrak{b})$, then $\mathfrak{a} \leqslant \mathfrak{b}$ if and only if $\left(\mathfrak{a}^{-1}\right)^{-1} \supseteq \mathfrak{b}$.

Corollary 1. For all $\mathfrak{a} \in \mathfrak{R}(\mathfrak{b}), \mathfrak{a} \sim\left(\mathfrak{a}^{-1}\right)^{-1}$ and for all $\mathfrak{b} \in \mathfrak{R}(\mathfrak{b}), \mathfrak{a} \sim \mathfrak{b}$ implies that $\left(\mathfrak{a}^{-1}\right)^{-1} \supseteq \mathfrak{b}$.

From now on, for all $\mathfrak{a} \in \mathfrak{R}(\mathfrak{b})$, we will denote $\left(\mathfrak{a}^{-1}\right)^{-1}$ by $\mathfrak{a}^{*}$. Of course, if $\mathfrak{a}$ is invertible, $\mathfrak{a}=\mathfrak{a}^{*}$; this is true in particular for the principal $\mathfrak{D}$-ideals of $\mathfrak{D}$ generated by regular elements.

Corollary 2. If $\mathfrak{a} \in \mathfrak{R}(\mathfrak{b})$, then $\mathfrak{a} \geqslant \mathfrak{0}$ if and only if $\mathfrak{a} \subseteq \mathfrak{b}$. 
Corollary 3. If $\left\{\mathfrak{a}_{i}\right\}_{i \in I}$ is a set of elements of $\mathcal{R}(\mathfrak{D})$, then

$$
\overline{\sum_{i \in I} \mathfrak{a}_{i}} \text { and } \overline{\bigcap_{i \in I} \mathfrak{a}_{i}{ }^{*}}
$$

are, respectively, the greatest lower bound and least upper bound of $\left\{\bar{a}_{i}\right\}_{i \in I}$ in $\overline{\mathfrak{R}(\mathfrak{D})}$.

Since the relation of quasi-divisibility is a reflexive, transitive and multiplicative relation on $\mathfrak{R}(\mathfrak{D})$, it is evident that if we define the relation $R_{\mathfrak{b}}$ on $\mathfrak{D}$ as follows: for all $a, b \in \mathfrak{D}, a R_{\mathbb{0}} b$ if and only if $(a) \leqslant(b)$, this relation is reflexive, transitive and multiplicative. Then, if $a, b, c \in \mathfrak{D}$ and if $a R_{0} b$ and $a R_{0} c$, then $(a) \leqslant(b)$ and $(a) \leqslant(c)$, which implies that $(a) \leqslant(b)+(c)$. But since

$$
b-c \in(b)+(c),(b-c) \geqslant(b)+(c) \geqslant(a)
$$

so that $a R_{\mathfrak{v}}(b-c)$. Therefore, $R_{\mathfrak{v}}$ is a subvaluation of $\mathfrak{D}$.

Proposition 3. If $\mathfrak{a}, \mathfrak{b}$ and $\mathfrak{c}$ are ideals of $\mathfrak{b}$, if $\mathfrak{a}+\mathfrak{b} \sim \mathfrak{D}$ and if $\mathfrak{a}+\mathfrak{c} \sim \mathfrak{b}$, then $\mathfrak{a}+\mathfrak{b} \mathfrak{c} \sim \mathfrak{D}$.

Proposition 4. If $\mathfrak{a}$ and $\mathfrak{b}$ are ideals of $\mathfrak{b}$ and if $\mathfrak{a}+\mathfrak{b} \sim \mathfrak{b}$, then $\mathfrak{a} \cap \mathfrak{b} \sim \mathfrak{a} \mathfrak{b}$.

Proposition 5. If $\mathfrak{a} \in \mathfrak{R}(\mathfrak{D})$ and if $\overline{\mathfrak{a}}$ is invertible in $\overline{\mathfrak{R}(\mathfrak{D})}$, then $\overline{\mathfrak{a}^{-1}}$ is the inverse of $\overrightarrow{\mathfrak{a}}$.

Proposition 6. If $\mathfrak{a}, b \in \mathfrak{R}(\mathfrak{b})$, if $\overline{\mathfrak{b}}$ is invertible in $\overline{\mathfrak{R}(\mathfrak{b})}$ and if $\mathfrak{a}: \mathfrak{b}$ denotes the set of all $a \in \mathfrak{S}$ such that $a \mathfrak{b} \subseteq \mathfrak{a}$, then $\mathfrak{a}: \mathfrak{b} \sim \mathfrak{a b}^{-1}$.

Definition. We will say that an $\mathfrak{D}$-ideal $\mathfrak{a}$ of $\mathfrak{D}$ is regular, if

1. a contains a regular element (of $\mathfrak{D}$ or of $\mathfrak{D}$, both statements are equivalent),

2. there is a regular element $a$ (in $\mathfrak{S}$ or in $\mathfrak{D}$, both statements are equivalent) such that $a \mathfrak{a} \subseteq \mathbb{D}$, i.e. $\mathfrak{a}^{-1}$ contains a regular element.

Let $\mathfrak{F}(\mathfrak{D})$ denote the set of all regular $\mathfrak{D}$-ideals of $\mathfrak{D}$. Then $\mathfrak{F}(\mathfrak{D})$ has the following properties:

1. $\mathfrak{F}(\mathfrak{D})$ is closed under multiplication.

2. $\mathfrak{D} \in \mathfrak{F}(\mathfrak{D})$.

3. If $\mathfrak{a} \in \mathfrak{F}(\mathfrak{D})$, then $\mathfrak{a}^{-1} \subseteq \mathfrak{F}(\mathfrak{D})$.

4. If $\mathfrak{a}, \mathfrak{b} \in \mathfrak{F}(\mathfrak{b})$, then $\mathfrak{a}+\mathfrak{b} \in \mathfrak{F}(\mathfrak{b})$ and $\mathfrak{a} \cap \mathfrak{b} \in \mathfrak{F}(\mathfrak{b})$.

5. If $\left\{\mathfrak{a}_{i}\right\}_{\imath \in I}$ is a set of elements of $\mathfrak{F}(\mathfrak{D})$ and if this set has an upper bound in $\mathfrak{F}(\mathfrak{D})$, then

$$
\sum a_{i} \in \mathfrak{F}(\mathfrak{D}),
$$

while if this set has a lower bound in $\mathfrak{F}(\mathfrak{D})$, then

$$
\cap \mathfrak{a}_{i} \in \mathfrak{F}(\mathfrak{D}) \text {. }
$$

Since the distributive law is valid on $\mathfrak{F}(\mathfrak{D}), \mathfrak{F}(\mathfrak{D})$ is a complete lattice-ordered semi-group (1, p. 201). 
Let us denote by $\overline{\mathfrak{F}(\mathfrak{b})}$, the set of all $\overline{\mathfrak{a}}$, where $\mathfrak{a} \in \mathfrak{F}(\mathfrak{o})$. We may notice that although $\mathfrak{a} \notin \mathfrak{F}(\mathfrak{D})$, it may very well be that $\mathfrak{a}^{-1} \in \mathfrak{F}(\mathfrak{D})$ and therefore, $\overline{\mathfrak{a}} \in \overline{\mathfrak{F}(\mathfrak{b})}$. It can easily be shown that $\overline{\mathfrak{F}(\mathfrak{D})}$ is also a complete lattice-ordered semi-group.

Definition. An element $a \in \mathfrak{D}$ is said to depend almost integrally on $\mathfrak{D}$ if there exists a regular element $b \in \mathfrak{D}$ such that $b a^{n} \in \mathfrak{D}$ for all natural integers $n$. If every element of $\mathfrak{D}$ that depends almost integrally on $\mathfrak{D}$ is in $\mathfrak{D}$, then $\mathfrak{D}$ is said to be fully integrally closed in $\mathfrak{D}$.

If $\mathfrak{D}$ is fully integrally closed in $\mathfrak{D}$ and if the ascending chain condition is valid for the ideals of $\mathfrak{D}$, then $\mathfrak{D}$ is integrally closed in $\mathfrak{D}$, that is, every element of $\mathfrak{D}$ which is a root of a monic polynomial with coefficients in $\mathfrak{D}$, is in $\mathfrak{D}$.

THEOREM 1. If $\mathfrak{D}$ is an order of $\mathfrak{D}$, then $\overline{\mathfrak{F}(\mathfrak{D})}$ is a group if and only if $\mathfrak{D}$ is fully integrally closed in $\mathfrak{S}$.

4. Definition and elementary Properties of Factorization Rings. Let $\mathfrak{o}$ be an order of $\mathfrak{D}$ which is fully integrally closed in $\mathfrak{D}$ so that $\overline{\mathfrak{F}(\mathfrak{o})}$ is a group. From the theory of partially ordered groups, we know that a unique factorization theorem is valid for the elements of $\overline{\mathfrak{F}(\mathfrak{D})}$ if and only if $\overline{\mathfrak{F}(\mathfrak{D})}$ satisfies the chain condition, that is, if $\overline{\mathfrak{a}}_{1} \geq \overline{\mathfrak{a}}_{2} \geq \overline{\mathfrak{a}}_{3}>\ldots$ is a properly descending chain of elements of $\overline{\mathfrak{F}(\mathfrak{D})}$, where $\overline{\mathfrak{a}}_{1} \geqslant \overline{\mathfrak{D}}$ for all indices $i$, then this chain is finite. When $\overline{\mathfrak{F}(\mathfrak{D})}$ satisfies this condition, then we will say that $\mathrm{D}$ is a factorization ring. Let us recall that an element $\overline{\mathfrak{p}}$ of $\overline{\mathfrak{F}(\mathfrak{D})}$ is a prime element if and only if $\overline{\mathfrak{p}}>\overline{\mathfrak{b}}$ and $\overline{\mathfrak{p}}>\overline{\mathfrak{a}} \geqslant \overline{\mathfrak{b}}$ implies that $\overline{\mathfrak{a}}=\overline{\mathfrak{b}}$ (one may say "for all $\overline{\mathfrak{a}} \in \overline{\mathfrak{F}(\mathfrak{b})}$ " "or "for all $\overline{\mathfrak{a}} \in \overline{\mathfrak{Q}(\mathfrak{D})}$," since $\overline{\mathfrak{p}}>\overline{\mathfrak{a}} \geqslant \overline{\mathfrak{D}}$ and $\overline{\mathfrak{p}} \in \overline{\mathfrak{F}(\mathfrak{D})}$ imply that $\overline{\mathfrak{a}} \in \overline{\mathfrak{F}(\mathfrak{p})})$, and that the unique factorization theorem states that if $\overline{\mathfrak{a}} \in \overline{\mathfrak{F}(\mathfrak{d})}$, then

$$
\overline{\mathfrak{a}}=\overline{\mathfrak{p}}_{1}^{m_{1}} \overline{\mathfrak{p}}_{2}^{m_{2}} \ldots \overline{\mathfrak{p}}_{r}^{m_{r}}
$$

where the $\overline{\mathfrak{p}}_{\imath}$ are prime elements of $\overline{\mathfrak{F}(\mathfrak{D})}$, this decomposition being unique, and $\overline{\mathfrak{a}} \geqslant \overrightarrow{\mathfrak{b}}$ if and only if $n_{i} \geqslant 0$ for all indices $i$.

Throughout this section, we assume that $\mathfrak{D}$ is a factorization ring with $\mathfrak{D}$ as full ring of quotients.

THEOREM 2. If $\overline{\mathfrak{p}}$ is a prime element of $\overline{\mathfrak{F}(\mathfrak{b})}$ and if $\mathfrak{p}=\mathfrak{p}^{*}$, then $\mathfrak{p}$ is a regular prime ideal of $\mathrm{D}$. We will call these prime ideals the relevant prime ideals of $\mathrm{o}$.

Proof. If $a, b \in \mathfrak{D}, a b \in \mathfrak{p}$ and $b \notin \mathfrak{p}$, then $(b)+\mathfrak{p} \supset \mathfrak{p}=\mathfrak{p}^{*}$ so that $\overline{\mathfrak{p}}>\overline{(b)+\mathfrak{p}} \geqslant \overline{\mathfrak{p}}$ and therefore, $(b)+\mathfrak{p} \sim \mathfrak{p}$. Then, $(a)=a \mathfrak{0} \sim a((b)+\mathfrak{p})$ $=(a b)+a \mathfrak{p} \subseteq \mathfrak{p}$ so that $(a) \geqslant \mathfrak{p}$ and therefore, $a \in \mathfrak{p}^{*}=\mathfrak{p}$. Since $\overline{\mathfrak{p}} \in \overline{\mathfrak{F}(\mathfrak{p})}$ and $\mathfrak{p}=\mathfrak{p}^{*}, \mathfrak{p}$ must contain a regular element of $\mathfrak{p}$.

Lemma 2. If $\mathfrak{p}$ is a relevant prime ideal of $\mathfrak{b}$ and if $\mathfrak{a}$ and $\mathfrak{b}$ are ideals of $\mathfrak{b}$, then, 
1. if $\mathfrak{a} \geqslant \mathfrak{p}^{m}, \mathfrak{a} \geqslant \geqslant \mathfrak{p}^{m+1}, \mathfrak{b} \geqslant \mathfrak{p}^{n}$ and $\mathfrak{b} \geqslant \geqslant \mathfrak{p}^{n+1}$, where $m$ and $n$ are non-negative integers, then $\mathfrak{a} \mathfrak{b} \geqslant \mathfrak{p}^{m+n}$ and $\mathfrak{a b} \geqslant \geqslant \mathfrak{p}^{m+n+1}$.

2. if for all natural numbers $n, \mathfrak{a} \geqslant \mathfrak{p}^{n}$, then for all $n, \mathfrak{a} \mathfrak{b} \geqslant \mathfrak{p}^{n}$.

Proof. First of all, let us suppose that $\mathfrak{a}$ and $\mathfrak{b}$ satisfy the hypothesis of the first case. Then surely, $\mathfrak{a} \mathfrak{b} \geqslant \mathfrak{p}^{m+n}$. If $\mathfrak{a} \mathfrak{b} \geqslant \mathfrak{p}^{m+n+1}$, then $\left(\mathfrak{a} \mathfrak{p}^{-m}\right)\left(\mathfrak{b}^{-n}\right) \geqslant \mathfrak{p}$ so that $\left(\mathfrak{a} \mathfrak{p}^{-m}\right)\left(\mathfrak{b p}^{-n}\right) \subseteq \mathfrak{p}$. But since $\mathfrak{a \mathfrak { p } ^ { - m }} \geqslant \mathfrak{o}$ and $\mathfrak{b p}^{-n} \geqslant \mathfrak{b}, \mathfrak{a p}^{-m}$ and $\mathfrak{b} \mathfrak{p}^{-n}$ are ideals of $\mathfrak{p}$ so that $\mathfrak{a p}^{-m} \subseteq \mathfrak{p}$ or $\mathfrak{b} \mathfrak{p}^{-n} \subseteq \mathfrak{p}$ which implies that $\mathfrak{a} \geqslant \mathfrak{p}^{m+1}$ or $\mathfrak{b} \geqslant \mathfrak{p}^{n+1}$, contradicting our hypotheses.

The second case is trivial since $\mathfrak{a} \mathfrak{b} \subseteq \mathfrak{a}$ and therefore, $\mathfrak{a} \mathfrak{b} \geqslant \mathfrak{a}$.

Now, if $\mathfrak{p}$ is a relevant prime ideal of $\mathfrak{b}$, we may use $\mathfrak{p}$ to define a valuation $V$ on $\mathfrak{D}$ as follows: if $a \in \mathfrak{D}$ and if there is a non-negative integer $n$ such that $(a) \geqslant \mathfrak{p}^{n}$ and $(a) \geqslant \geqslant \mathfrak{p}^{n+1}$, we set $V(a)=n$, while if for all natural numbers $n$, $(a) \geqslant \mathfrak{p}^{n}$, we set $V(a)=\infty$. By Lemma 2 , for all $a, b \in \mathfrak{D}, V(a b)=V(a)$ $+V(b)$. Now, if $a, b \in \mathfrak{D}$ and if $n$ is a non-negative integer such that $(a) \geqslant \mathfrak{p}^{n}$ and $(b) \geqslant \mathfrak{p}^{n}$, then since $(a-b) \subseteq(a)+(b)$, we have

$$
\overline{(a-b)} \geqslant \overline{(a)+(b)} \geqslant \overline{\mathfrak{p}}^{n}
$$

(Cor. 3, Prop. 2) so that $V(a-b) \geqslant \min \{V(a), V(b)\}$. If $a$ is a regular element of $\mathfrak{o}$, since $(a) \in \mathfrak{F}(\mathfrak{o})$, it is evident that $V(a) \neq \infty$. We may then extend the function $V$ to all of $\mathfrak{D}$ as follows: if $a \in \mathfrak{D}, a=b / c$, where $b, c \in \mathfrak{D}$ and $c$ is regular, and we set $V(a)=V(b)-V(c)$. Then, one can easily verify that $V$ is well defined on all of $\mathfrak{S}$ and that it satisfies $\mathrm{V}^{\prime}$ and $\mathrm{V} 2$. If $n$ is any nonnegative integer, since $\left(\mathfrak{p}^{n+1}\right)^{*} \subset\left(\mathfrak{p}^{n}\right)^{*}$, there is an element $a \in\left(\mathfrak{p}^{n}\right)^{*}$ such that $a \notin\left(\mathfrak{p}^{n+1}\right)^{*}$ and therefore, $V(a)=n$. Evidently, $V(0)=\infty$. Now $\mathfrak{p}$ contains a regular element $a$, and therefore, $V(a)=m>0$, and if $n$ is any positive integer, one can find an integer $k$ such that $V\left(a^{k}\right)=k m \geqslant n$ and therefore, there exists an element $b \in \mathfrak{D}$ such that $V(b)=k m-n$ so that

$$
V\left(b a^{-k}\right)=V(b)-V\left(a^{k}\right)=-n .
$$

Thus $V$ is a special valuation of $\mathfrak{D}$. We will say that $V$ is the valuation of $\mathfrak{D}$ determined by the relevant prime ideal $\mathfrak{p}$ of $\mathfrak{D}$.

THEOREM 3. If $\mathfrak{p}$ is a relevant prime ideal of $\mathfrak{D}$ and if $V$ is the valuation of $\mathfrak{D}$ determined by $\mathfrak{p}$, then for each positive integer $n$,

$$
\mathfrak{p}^{(n)}=\left(\mathfrak{p}^{n}\right)^{*}=\{a \in \mathfrak{p} \mid V(a) \geqslant n\} .
$$

Proof. If $a \in \mathfrak{D}, V(a) \geqslant n$ if and only if $(a) \geqslant \mathfrak{p}^{n}$, which evidently means that $a \in\left(\mathfrak{p}^{n}\right)^{*}$, so that

$$
\left(\mathfrak{p}^{n}\right)^{*}=\{a \in \mathfrak{D} \mid V(a) \geqslant n\} .
$$

Then, by Lemma 1 , each $\left(\mathfrak{p}^{n}\right)^{*}$ is a $\mathfrak{p}$-primary ideal of $\mathfrak{o}$ and since $\mathfrak{p}^{n} \subseteq\left(\mathfrak{p}^{n}\right)^{*}$, $\mathfrak{p}^{(n)} \subseteq\left(\mathfrak{p}^{n}\right)^{*}$. If $a \in\left(\mathfrak{p}^{n}\right)^{*}$, then $(a) \geqslant \mathfrak{p}^{n}$ and therefore, $a\left(\mathfrak{p}^{n}\right)^{-1} \subseteq \mathfrak{o}$. Since $\left(\mathfrak{p}^{n}\right)^{-1} \mathfrak{p}^{n} \sim \mathfrak{p}$, there exists $b \in\left(\mathfrak{p}^{n}\right)^{-1} \mathfrak{p}^{n}$ such that $b \notin \mathfrak{p}$. Then 


$$
a b \in a\left(\left(\mathfrak{p}^{n}\right)^{-1} \mathfrak{p}^{n}\right)=\left(a\left(\mathfrak{p}^{n}\right)^{-1}\right) \mathfrak{p}^{n} \subseteq \mathfrak{p}^{n}
$$

so that $a \in\left(\mathfrak{p}^{n}\right)_{\mathfrak{p}}=\mathfrak{p}^{(n)}$ and therefore, $\mathfrak{p}^{(n)}=\left(\mathfrak{p}^{n}\right)^{*}$.

Under the hypotheses of Theorem 3 , if we set

$$
\mathfrak{p}^{\prime}=\{a \in \mathfrak{o} \mid V(a)=\infty\}
$$

by Lemma $1, \mathfrak{p}^{\prime}$ is a prime ideal of $\mathfrak{D}$ and

$$
\mathfrak{p}^{\prime}=\bigcap_{n=1}^{\infty} \mathfrak{p}^{(n)}
$$

We will call $\mathfrak{p}^{\prime}$ the associate of $\mathfrak{p}$.

THEOREM 4. If $\mathfrak{p}$ is a relevant prime ideal of $\mathfrak{b}$ and if $\mathfrak{p}^{\prime}$ is the associate of $\mathfrak{p}$, then $\mathfrak{p}^{\prime}$ has the following properties:

1. for every natural integer $n, \mathfrak{p}^{\prime} \geqslant \mathfrak{p}^{n}$ and $\mathfrak{p}^{\prime}$ contains any $\mathfrak{D}$-ideal of $\mathfrak{D}$ that has this property,

2. $(\mathfrak{p})^{\prime *}=\mathfrak{p}^{\prime}$.

3. any prime ideal of $\mathfrak{D}$ that is properly contained in $\mathfrak{p}$, is contained in $\mathfrak{p}^{\prime}$.

Proof. If $n$ is any natural integer, for all $a \in \mathfrak{p}^{\prime},(a) \geqslant \mathfrak{p}^{n}$, so that by Corollary 3 of Proposition 2,

$$
\overline{\mathfrak{p}^{\prime}}=\overline{\sum_{a \in \mathfrak{p}^{\prime}}(a)} \geqslant \overline{\mathfrak{p}^{n}} .
$$

Then, if $\mathfrak{a}$ is any $\mathfrak{b}$-ideal of $\mathfrak{D}$ with the property that for all natural integers $n$, $\mathfrak{a} \geqslant \mathfrak{p}^{n}$, for all $a \in \mathfrak{a},(a) \geqslant \mathfrak{a} \geqslant \mathfrak{p}^{n}$ so that $V(a)=\infty$ and therefore, $a \in \mathfrak{p}^{\prime}$ and $\mathfrak{a} \subseteq \mathfrak{p}^{\prime}$. In particular, $\left(\mathfrak{p}^{\prime}\right)^{*}$ has this property, so that $\left(\mathfrak{p}^{\prime}\right)^{*}=\mathfrak{p}^{\prime}$.

Let $\mathfrak{a}$ be an ideal of $\mathfrak{b}$ such that $\mathfrak{a} \subset \mathfrak{p}$ and $\mathfrak{a} \not \subset \mathfrak{p}^{\prime}$. Then, there is a nonnegative integer $n$ such that $\mathfrak{a} \geqslant \mathfrak{p}^{n}$ and $\mathfrak{a} \triangleq \geqslant \mathfrak{p}^{n+1}$. Now $\left(\mathfrak{a} \mathfrak{p}^{-1}\right) \mathfrak{p}=\mathfrak{a}\left(\mathfrak{p}^{-1} \mathfrak{p}\right) \subseteq \mathfrak{a}$. Since $\mathfrak{a} \subset \mathfrak{p}, \mathfrak{p} \not \subset \mathfrak{a}$ and $\mathfrak{a} \mathfrak{p}^{-1} \geqslant \mathfrak{0}$ so that $\mathfrak{a} \mathfrak{p}^{-1} \subseteq \mathfrak{D}$. If $\mathfrak{a} \mathfrak{p}^{-1} \subseteq \mathfrak{a}$, then $\mathfrak{a} \mathfrak{p}^{-1}$ $\geqslant a \geqslant \mathfrak{p}^{n}$ so that $a \geqslant \mathfrak{p}^{n+1}$, a contradiction. Therefore, $\mathfrak{a}$ is not a prime ideal of $\mathrm{D}$.

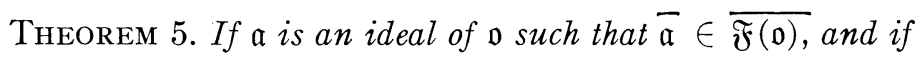

$$
\overline{\mathfrak{a}}={\overline{\mathfrak{p}_{1}}}^{{ }^{n}}{\overline{\mathfrak{p}_{2}}}^{n_{2}} \ldots{\overline{\mathfrak{p}_{r}}}^{{ }^{r}}
$$

where $\mathfrak{p}_{1}, \mathfrak{p}_{2}, \ldots, \mathfrak{p}_{r}$ are relevant prime ideals of $\mathfrak{D}$, then

$$
\mathfrak{a}^{*}=\mathfrak{p}_{1}^{\left(n_{1}\right)} \cap \mathfrak{p}_{2}^{\left(n_{2}\right)} \cap \ldots \cap \mathfrak{p}_{r}^{\left(n_{r}\right)} .
$$

Proof. Since $\overline{\mathfrak{a}}$ is the least upper bound, in $\overline{\mathfrak{F}(\mathfrak{D})}$, and therefore also in $\overline{\mathfrak{l}(\mathfrak{D})}$, of the set

$$
\left\{\overline{p_{1}^{n_{1}}}, \overline{p_{2}^{n_{2}}}, \ldots, \overline{p_{r}^{n_{r}}}\right\}
$$

by Corollary 3 of Proposition 2,

$$
\mathfrak{a} \sim \mathfrak{p}_{1}^{\left(n_{1}\right)} \cap \mathfrak{p}_{2}^{\left(n_{2}\right)} \cap \ldots \cap \mathfrak{p}_{r}^{\left(n_{r}\right)}
$$


and therefore,

$$
\mathfrak{a}^{*} \supseteq \mathfrak{p}_{1}^{\left(n_{1}\right)} \cap \mathfrak{p}_{2}^{\left(n_{2}\right)} \cap \ldots \cap \mathfrak{p}_{r}^{\left(n_{r}\right)}
$$

But since, for each $i=1,2, \ldots, r$,

$$
\mathfrak{a}^{*} \sim \mathfrak{a} \geqslant \mathfrak{p}_{i}^{n_{i}}, \mathfrak{a}^{*} \subseteq \mathfrak{p}_{i}^{\left(n_{i}\right)}
$$

and therefore,

$$
\mathfrak{a}^{*} \subseteq \mathfrak{p}_{1}^{\left(n_{1}\right)} \cap \mathfrak{p}_{2}^{\left(n_{2}\right)} \cap \ldots \cap \mathfrak{p}_{r}^{\left(n_{r}\right)}
$$

COROLlary 1. If $a$ is a regular element of $\mathfrak{b}$ and if $a$ is not a unit of $\mathfrak{b}$, then

$$
(a)=\mathfrak{p}_{1}^{\left(n_{1}\right)} \cap \mathfrak{p}_{2}^{\left(n_{2}\right)} \cap \ldots \cap \mathfrak{p}_{r}^{\left(n_{r}\right)}
$$

where $\mathfrak{p}_{1}, \mathfrak{p}_{2}, \ldots, \mathfrak{p}_{r}$ are relevant prime ideals of $\mathfrak{D}$.

COROLlary 2. The relevant prime ideals of $\mathrm{o}$ are just the minimal proper regular prime ideals of $\mathfrak{D}$. Every regular prime ideal of $\mathbf{D}$ contains a relevant prime ideal of $\mathfrak{D}$ so that the non-minimal regular prime ideals of $\mathfrak{D}$ are all quasi-equal to $\mathbf{b}$.

Proof. Since the relevant prime ideals of $\mathfrak{b}$ are regular and since no relevant prime ideal is properly contained in another, all we have to show is that any regular prime ideal $\mathfrak{p}$ of $\mathfrak{D}$ contains a relevant prime ideal of $\mathfrak{D}$.

We need only consider the case where $\mathfrak{p} \neq \mathfrak{0}$, and then, $\mathfrak{p}$ contains a regular element $a$ which is not a unit of $\mathfrak{b}$, so that by Corollary 1 of Theorem 5 ,

$$
(a)=\mathfrak{p}_{1}^{\left(n_{1}\right)} \cap \mathfrak{p}_{2}^{\left(n_{2}\right)} \cap \ldots \cap \mathfrak{p}_{r}^{\left(n_{r}\right)}
$$

where $\mathfrak{p}_{1}, \mathfrak{p}_{2}, \ldots, \mathfrak{p}_{r}$ are relevant prime ideals of $\mathfrak{p}$, and evidently, $\mathfrak{p}$ must contain one of them.

THEOREM 6. If $\mathfrak{p}$ is a relevant prime ideal of $\mathfrak{b}$, then the only $\mathfrak{p}$-primary ideals of $\mathfrak{p}$ are the formal powers of $\mathfrak{p}$.

Proof. Let $\mathfrak{q}$ be a $\mathfrak{p}$-primary ideal of $\mathfrak{p}$. Since $\mathfrak{p}$ contains a regular element $a$ and since $\operatorname{rad} \mathfrak{q}=\mathfrak{p}, a^{m} \in \mathfrak{q}$ for some positive integer $m$ and $\mathfrak{q}$ is regular. Since $a^{m}$ is not a unit of $\mathfrak{D}$, by Corollary 1 of Theorem 5 ,

$$
\left(a^{m}\right)=p_{1}^{\left(n_{1}\right)} \cap \mathfrak{p}_{2}^{\left(n_{2}\right)} \cap \ldots \cap \mathfrak{p}_{r}^{\left(n_{r}\right)}
$$

where $\mathfrak{p}_{1}, \mathfrak{p}_{2}, \ldots, \mathfrak{p}_{r}$ are relevant prime ideals of $\mathfrak{o}$, and $\mathfrak{p}$ must contain one of these, say $\mathfrak{p} \supseteq \mathfrak{p}_{1}$. Since $\mathfrak{p}$ is relevant, $\mathfrak{p}=\mathfrak{p}_{1}$. Then $\mathfrak{p}^{\left(n_{1}\right)}=\left(a^{m}\right)_{\mathfrak{p}}$ and since $\mathfrak{q}$ is a $\mathfrak{p}$-primary ideal containing $\left(a^{m}\right)$,

$$
\mathfrak{q} \supseteq \mathfrak{p}^{\left(n_{1}\right)}
$$

so that

$$
\mathfrak{q} \leqslant \mathfrak{p}^{n_{1}}
$$


and therefore, $\mathfrak{q} \sim \mathfrak{p}^{n}$ for some positive integer $n \leqslant n_{1}$ and $\mathfrak{q} \subseteq \mathfrak{p}^{(n)}$. Then, by Proposition 6 ,

$$
\mathfrak{q}: \mathfrak{p}^{(n)} \sim \mathfrak{q}\left(\mathfrak{p}^{(n)}\right)^{-1} \sim \mathfrak{q}\left(\mathfrak{p}^{n}\right)^{-1} \sim \mathfrak{D}
$$

so that there exists an element $b \in \mathfrak{q}: \mathfrak{p}^{(n)}$ such that $b \notin \mathfrak{p}$. Then, $b \mathfrak{p}^{(n)} \subseteq \mathfrak{q}$, and since $b \notin \mathfrak{p}$ and $\mathfrak{q}$ is $\mathfrak{p}$-primary, $\mathfrak{p}^{(n)} \subseteq \mathfrak{q}$.

Corollary. If $\mathfrak{a}$ is an ideal of $\mathfrak{D}$ and if

$$
\infty \neq n=\min \{V(a) \mid a \in \mathfrak{a}\}
$$

then $\mathfrak{a}_{\mathfrak{p}}=\mathfrak{p}^{(n)}$ (by definition, $\left.\mathfrak{p}^{(0)}=\mathfrak{p}\right)$.

Proof. It is evident that $\mathfrak{a} \subseteq \mathfrak{p}^{(n)}$ and that $\left.\mathfrak{a} \not \subset \mathfrak{p}^{(n+1}\right)$. If $\mathfrak{p}$ were not a minimal prime ideal of $\mathfrak{a}$, then $\mathfrak{p}$ would properly contain a minimal prime ideal of $\mathfrak{a}$, which, by Theorem 4 , would be contained in $\mathfrak{p}^{\prime}$ so that $\mathfrak{a} \subseteq \mathfrak{p}^{\prime}$, a contradiction. Therefore, $\mathfrak{p}$ is a minimal prime ideal of $\mathfrak{a}$ and $\mathfrak{a}_{\mathfrak{p}}$ is $\mathfrak{p}$-primary. Then, since $\mathfrak{a} \subseteq \mathfrak{p}^{(n)}, \mathfrak{a}_{\mathfrak{p}} \subseteq \mathfrak{p}^{(n)}$ and since $\mathfrak{a} \subseteq \mathfrak{a}_{\mathfrak{p}}, \mathfrak{a}_{\mathfrak{p}} \not \subset \mathfrak{p}^{(n+1)}$ so that by Theorem 6 , $\mathfrak{a}_{\mathfrak{p}}=\mathfrak{p}^{(n)}$.

THEOREM 7. If $\mathfrak{p}$ is a relevant prime ideal of $\mathfrak{D}$, if $\mathfrak{p}^{\prime}$ is the associate of $\mathfrak{p}$ and if $V$ is the valuation of $\mathfrak{D}$ determined by $\mathfrak{p}$, then $\left(\mathfrak{o} / \mathfrak{p}^{\prime}\right)_{\left(\mathfrak{p} / \mathfrak{p}^{\prime}\right)}$ is a regular local ring of dimension 1 (valuation ring determined by a discrete, rank 1 valuation of its field of quotients) and if $\phi$ denotes the natural homomorphism of $\mathfrak{D}$ onto $\mathfrak{D} / \mathfrak{p}^{\prime}$, then, for any two elements $a, b \in \mathfrak{D}, V(a) \leqslant V(b)$ if and only if $\phi(a)$ divides $\phi(b)$ in $\left(\mathfrak{o} / \mathfrak{p}^{\prime}\right)_{\left(\mathfrak{p} / \mathfrak{p}^{\prime}\right)}$.

Proof. Since $\mathfrak{p}^{\prime}$ is a prime ideal of $\mathfrak{o}, \mathfrak{o} / \mathfrak{p}^{\prime}$ and $\left(\mathfrak{o} / \mathfrak{p}^{\prime}\right)_{\left(\mathfrak{p} / \mathfrak{p}^{\prime}\right)}$ are integral domains. Since every prime ideal of $\mathfrak{o}$ that is properly contained in $\mathfrak{p}$ must be contained in $\mathfrak{p}^{\prime}$ (Theorem 4 ), the null ideal is the only prime ideal of $\mathfrak{o} / \mathfrak{p}^{\prime}$ that is properly contained in $\mathfrak{p} / \mathfrak{p}^{\prime}$ so that $\left(\mathfrak{o} / \mathfrak{p}^{\prime}\right)_{\left(\mathfrak{p} / \mathfrak{p}^{\prime}\right)}$ contains only one non-trivial prime ideal. Then, an arbitrary non-trivial ideal of $\left(\mathfrak{D} / \mathfrak{p}^{\prime}\right)_{\left(\mathfrak{p} / \mathfrak{w}^{\prime}\right)}$ must be a primary ideal belonging to this unique non-trivial prime ideal. Now, the only p-primary ideals of $\mathfrak{p}$ are the formal powers of $\mathfrak{p}$ (Theorem 6 ) and they are totally ordered under ordinary inclusion and all contain $\mathfrak{p}^{\prime}$ so that the $\mathfrak{p} / \mathfrak{p}^{\prime}$-primary ideals of $\mathfrak{b} / \mathfrak{p}^{\prime}$ are totally ordered under ordinary inclusion and therefore, the nontrivial ideals of $\left(\mathfrak{o} / \mathfrak{p}^{\prime}\right)_{\left(\mathfrak{p} / \mathfrak{p}^{\prime}\right)}$ are also totally ordered under ordinary inclusion. Thus, $\left(\mathfrak{o} / \mathfrak{p}^{\prime}\right)_{\left(\mathfrak{p} / \mathfrak{p}^{\prime}\right)}$ is a regular local ring of dimension 1 .

If $a, b \in \mathfrak{D}, V(a) \leqslant V(b)$ if and only if for all non-negative integers $n$, $(a) \geqslant \mathfrak{p}^{n}$ implies that $(b) \geqslant \mathfrak{p}^{n}$, that is, $a \in \mathfrak{p}^{(n)}$ implies that $b \in \mathfrak{p}^{(n)}$ or $\phi(a)$ $\in \phi\left(\mathfrak{p}^{(n)}\right)$ implies that $\phi(b) \in \phi\left(\mathfrak{p}^{(n)}\right)$, which evidently means that $\phi(a)$ divides $\phi(b)$ in $\left(\mathfrak{b} / \mathfrak{p}^{\prime}\right)_{\left(\mathfrak{p} / \mathfrak{p}^{\prime}\right)}$.

Remarks. If $\mathfrak{D}$ is an order of $\mathfrak{D}$, if $\mathfrak{D}$ is fully integrally closed in $\mathfrak{D}$ and if the ascending chain condition holds for the ideals of $\boldsymbol{D}$, then $\boldsymbol{D}$ is surely a factorization ring, for if $\overline{\mathfrak{a}_{1}}>\overline{\mathfrak{a}_{2}}>\overline{\mathfrak{a}_{3}}>\ldots$ is a chain of elements of $\overline{\mathfrak{F}(\mathfrak{b})}$ and if, for each index $i, \overline{\mathfrak{a}_{i}} \geqslant \overline{\mathfrak{b}}$, then the ascending chain $\mathfrak{a}_{1}{ }^{*} \subset \mathfrak{a}_{2}{ }^{*} \subset \mathfrak{a}_{3}{ }^{*} \subset \ldots$ of ideals of $\mathfrak{o}$ must be finite. Furthermore, by Krull's Intersection Theorem, 


$$
\mathfrak{p}^{\prime}=\bigcap_{n=1}^{\infty} \mathfrak{p}^{(n)}=(0)_{\mathfrak{p}}
$$

so that $\left(\mathfrak{D} / \mathfrak{p}^{\prime}\right)_{\left(\mathfrak{p} / \mathfrak{p}^{\prime}\right)}$ is just the generalized ring of quotients $\mathfrak{D}_{\mathfrak{p}}$ and $\mathfrak{p}^{\prime}$ is a minimal prime ideal of $\mathfrak{D}$. (We have thus obtained the results of $(\mathbf{5}, \S 4.7$ and $\$ 4.9$ ).)

Under these assumptions, since the associates of relevant prime ideals of $\mathfrak{b}$ are minimal prime ideals of $\mathfrak{b}$, there is only a finite number of them and by Theorem 4 , for each relevant prime ideal $\mathfrak{p}$ of $\mathfrak{D}, \mathfrak{p}^{\prime}$ is the only prime ideal of $\mathfrak{b}$ properly contained in $\mathfrak{p}$. If $\mathfrak{a}$ is an ideal of $\mathfrak{p}$ contained in $\mathfrak{p}^{\prime}$, then

$$
\mathfrak{p}^{\prime}=(0)_{\mathfrak{p}} \subseteq \mathfrak{a}_{\mathfrak{p}} \subseteq \mathfrak{p}_{\mathfrak{p}}{ }^{\prime}=\mathfrak{p}^{\prime}
$$

so that $\mathfrak{a}_{\mathfrak{p}}=\mathfrak{p}^{\prime}$. Therefore, $\mathfrak{p}^{\prime}$ is the only $\mathfrak{p}^{\prime}$-primary ideal of $\mathfrak{o}$, and if we set

$$
\mathfrak{p}^{\prime}=\bigcap_{n=1}^{\infty} \mathfrak{p}^{(n)}=\mathfrak{p}^{(\infty)}
$$

then we may restate the Corollary of Theorem 5 more generally as follows: If $\mathfrak{a}$ is an ideal of $\mathfrak{D}$ and if $n=\min \{V(a) \mid a \in \mathfrak{a}\} \quad(n$ may equal $\infty$ ), then $\mathfrak{a}_{\mathfrak{p}}=\mathfrak{p}^{(n)}$.

THEOREM 8. If $\left\{\mathfrak{p}_{i}\right\}_{i \in I}$ is the set of relevant prime ideals of $\mathfrak{D}$ and if for each $i \in I, V_{i}$ denotes the valuation of $\mathfrak{O}$ determined by $\mathfrak{p}_{i}$, then for all $a \in \mathfrak{O}$, $a \in \mathfrak{D}$ if and only if $V_{i}(a) \geqslant 0$ for all $i \in I$. Furthermore, if for each $i \in I$,

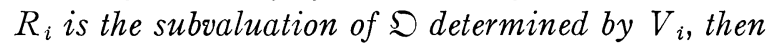

$$
R_{\mathfrak{v}}=\bigcap_{i \in I} R_{i}
$$

Proof. From the definition of $V_{i}$, it is evident that if $a \in \mathfrak{D}$, then $V_{i}(a) \geqslant 0$ for all $i \in I$. Conversely, let $a$ be an element of $\mathfrak{D}$ such that $V_{i}(a) \geqslant 0$ for all $i \in I$. Then, $a=b / c$, where $b, c \in \mathfrak{D}, c$ is regular and $V_{i}(b) \geqslant V_{i}(c)$ for all $i \in I$. If $c$ is not a unit of $\mathfrak{b}$, by Corollary 1 of Theorem 5 ,

$$
(c)=\mathfrak{p}_{i_{1}}^{\left(n_{1}\right)} \cap \mathfrak{p}_{i_{2}}^{\left(n_{2}\right)} \cap \ldots \cap \mathfrak{p}_{i_{r}}^{\left(n_{r}\right)}
$$

where $i_{1}, i_{2}, \ldots, i_{r} \in I$. Then, for each $j=1,2, \ldots, r$,

$$
\text { since } V_{i_{j}}(b) \geqslant V_{i_{j}}(c),(b) \geqslant \mathfrak{p}_{i_{j}}^{n_{j}} \text { so that } b \in \mathfrak{p}_{i_{j}}^{\left(n_{j}\right)}
$$

and therefore, $b \in(c)$ and $a=b / c \in \mathfrak{D}$. We may notice here that for every regular element $c \in \mathfrak{D}, V_{i}(c)=0$ for all $i \in I$ except a finite number.

If $a, b \in \mathfrak{0}$ and $a R_{0} b$, then $(a) \leqslant(b)$ and from the definition of $V_{i}$, it is evident that $V_{i}(a) \leqslant V_{i}(b)$. In general, if $a, b \in \mathfrak{D}, a=c_{1} / d_{1}, b=c_{2} / d_{2}$, where $c_{1}, d_{1}, c_{2}, d_{2} \in \mathfrak{D}$ and $d_{1}$ and $d_{2}$ are regular, and if $(a) \leqslant(b)$, then $\left(c_{1}\right)$ $\left(d_{1}\right)^{-1} \leqslant\left(c_{2}\right)\left(d_{2}\right)^{-1}$ so that $\left(c_{1} d_{2}\right) \leqslant\left(c_{2} d_{1}\right)$ and therefore,

$$
V_{i}\left(c_{1}\right)+V_{i}\left(d_{2}\right)=V_{i}\left(c_{1} d_{2}\right) \leqslant V_{i}\left(c_{2} d_{1}\right)=V_{i}\left(c_{2}\right)+V_{i}\left(d_{1}\right),
$$

and this implies that 


$$
V_{i}(a)=V_{i}\left(c_{1}\right)-V_{i}\left(d_{1}\right) \leqslant V_{i}\left(c_{2}\right)-V_{i}\left(d_{2}\right)=V_{i}(b),
$$

i.e. $a R_{i} b$. Therefore,

$$
R_{\mathfrak{o}} \subseteq \bigcap_{i \in I} R_{i}
$$

Conversely, if $a, b \in \mathfrak{D}$ and if for all $i \in I, a R_{i} b$, i.e. $V_{i}(a) \leqslant V_{i}(b)$, then, if $c \in(a)^{-1}, c a \in \mathrm{D}$ so that for all $i \in I$,

$$
V_{i}(c b)=V_{i}(c)+V_{i}(b) \geqslant V_{i}(c)+V_{i}(a)=V_{i}(c a) \geqslant 0
$$

and therefore, $c b \in \mathfrak{D}$. Therefore, $(a)^{-1} \subseteq(b)^{-1}$, i.e., $(a) \leqslant(b)$ or $a R_{\mathrm{o}} b$.

As a particular case of this theorem, we have that if there is only one relevant prime ideal $\mathfrak{p}$ in $\mathfrak{D}$ and if $V$ is the valuation of $\mathfrak{D}$ determined by $\mathfrak{p}$, then $\mathfrak{D}$ is the order of $\mathfrak{D}$ determined by $V$ and $R_{0}$ is the sub-valuation of $\mathfrak{D}$ determined by $V$.

5. Two characterizations of factorization rings. In this section, we will see that the properties of factorization rings given by Corollary 1 of Theorem 5 and by Theorem 8 may be used to characterize factorization rings.

If $\mathfrak{D}$ is an order of $\mathfrak{D}$ and if $V$ is a special non-negative valuation of $\mathfrak{O}$, we define the function $V_{\mathfrak{v}}$ on $\mathfrak{R}(\mathfrak{D})$ as follows: if $\mathfrak{a} \in \mathfrak{R}(\mathfrak{D})$ and if $\{V(a) \mid a \in \mathfrak{a}\}$ has a minimum, then $V_{\mathfrak{p}}(\mathfrak{a})=\min \{V(a) \mid a \in \mathfrak{a}\}$, while otherwise, $V_{\mathfrak{0}}(\mathfrak{a})=\infty^{\prime}$. It is evident that for all $a \in \mathfrak{O}, V_{\mathfrak{o}}((a))=V(a)$ so that we may think of $V_{\mathfrak{0}}$ as an extension of $V$ and drop the subscript $\mathfrak{D}$ where no ambiguity arises.

Lemma 3. If $\mathfrak{D}$ is an order of $\mathfrak{D}$ and if $V$ is a special valuation of $\mathfrak{D}$ such that $V(a) \geqslant 0$ for all $a \in \mathfrak{0}$, then $V_{0}$ is a homomorphism of $\mathbb{R}(\mathfrak{0})$ onto $G^{\prime \prime}$, and for all $\mathfrak{a}, \mathfrak{b} \in \mathfrak{R}(\mathfrak{b}), V(\mathfrak{a}+\mathfrak{b})=\min \{V(\mathfrak{a}), V(\mathfrak{b})\}$.

Proof. Let $\mathfrak{a}, \mathfrak{b} \in \mathfrak{R}(\mathfrak{o})$. It is evident that $\mathfrak{a} \subseteq \mathfrak{b}$ implies that $V(\mathfrak{a}) \geqslant V(\mathfrak{b})$. Any element of $\mathfrak{a} \mathfrak{b}$ has the form $a_{1} b_{1}+\ldots+a_{n} b_{n}$ where $a_{\mathfrak{i}} \in \mathfrak{a}$ and $b_{\mathfrak{i}} \in \mathfrak{b}$, and

$$
\begin{aligned}
V\left(a_{1} b_{1}+\ldots+a_{n} b_{n}\right) & \geqslant \min \left\{V\left(a_{1}\right)+V\left(b_{1}\right), \ldots, V\left(a_{n}\right)+V\left(b_{n}\right)\right\} \\
& \geqslant V(\mathfrak{a})+V(\mathfrak{b})
\end{aligned}
$$

so that $V(\mathfrak{a} \mathfrak{b}) \geqslant V(\mathfrak{a})+V(\mathfrak{b})$. To establish the reverse inequality, let us suppose first of all that $V(\mathfrak{a}) \neq \infty^{\prime} \neq V(\mathfrak{b})$ so that there exist $a \in \mathfrak{a}$ and $b \in \mathfrak{b}$ such that $V(\mathfrak{a})=V(a)$ and $V(\mathfrak{b})=V(b)$, and then,

$$
V(\mathfrak{a})+V(\mathfrak{b})=V(a)+V(b)=V(a b) \geqslant V(\mathfrak{a} \mathfrak{b}) .
$$

Secondly, let us suppose that $V(\mathfrak{a})=\infty^{\prime}$ and that $V(\mathfrak{b}) \neq \infty$. Then, there exists $b \in \mathfrak{b}$ such that $V(b) \neq \infty$ and if $n$ is any ordinary integer, there exists $a \in \mathfrak{a}$ such that $V(a) \leqslant n-V(b)$ so that $V(a b)=V(a)+V(b) \leqslant n$ and therefore, $V(\mathfrak{a} \mathfrak{b})=\infty^{\prime}=V(\mathfrak{a})+V(\mathfrak{b})$. Finally, if $V(\mathfrak{a})=\infty^{\prime}$ and $V(\mathfrak{b})=\infty$, for every $b \in \mathfrak{b}, V(b)=\infty$ so that for every $a \in \mathfrak{a}, V(a b)=V(a)+V(b)$ $=\infty$ and therefore, $V(\mathfrak{a} \mathfrak{b})=\infty=V(\mathfrak{a})+V(\mathfrak{b})$. That $V_{0}$ is a function of 
$\mathfrak{R}(\mathfrak{D})$ onto $G^{\prime \prime}$ is evident, since for all $a \in \mathfrak{D}, V((a))=V(a)$ and since $V(\mathfrak{D})$ $=\infty^{\prime}$. If $\mathfrak{a}, \mathfrak{b} \in \mathfrak{R}(\mathfrak{b})$, since $\mathfrak{a} \subseteq \mathfrak{a}+\mathfrak{b}, \quad V(\mathfrak{a}) \geqslant V(\mathfrak{a}+\mathfrak{b})$ and similarly, $V(\mathfrak{b}) \geqslant V(\mathfrak{a}+\mathfrak{b})$ so that $\min \{V(\mathfrak{a}), V(\mathfrak{b})\} \geqslant V(\mathfrak{a}+\mathfrak{b})$. Then, if $a \in \mathfrak{a}$ and $b \in \mathfrak{b}$,

$$
V(a+b) \geqslant \min \{V(a), V(b)\} \geqslant \min \{V(\mathfrak{a}), V(\mathfrak{b})\}
$$

so that $V(\mathfrak{a}+\mathfrak{b}) \geqslant \min \{V(\mathfrak{a}), V(\mathfrak{b})\}$.

LEMMA 4. If $\mathfrak{D}$ is a factorization ring with $\mathfrak{D}$ as full ring of quotients, if $W$ is a special valuation of $\mathfrak{D}$ such that for all $a \in \mathfrak{D}, W(a) \geqslant 0$, and if $\{a \in \mathfrak{o} \mid W(a)$ $>0\}$ is a relevant prime ideal $\mathfrak{p}$ of $\mathfrak{D}$, then $W$ coincides with the valuation $V$ of $\mathfrak{O}$ determined by $\mathrm{p}$.

Proof. By Lemma 1, for each positive integer $n$, the set

$$
\mathfrak{q}_{n}=\{a \in \mathfrak{D} \mid W(a) \geqslant n\}
$$

is a $\mathfrak{p}$-primary ideal of $\mathfrak{p}$. Since $\mathfrak{p}^{n} \subseteq \mathfrak{q}_{n}, \mathfrak{p}^{(n)} \subseteq \mathfrak{q}_{n}$ so that by Theorem $6, \mathfrak{q}_{n}=\mathfrak{p}^{(m)}$ where $1 \leqslant m \leqslant n$, and therefore, $W\left(\mathfrak{q}_{n}\right)=W\left(\mathfrak{p}^{(m)}\right)=W\left(\mathfrak{p}^{m}\right)=m W(\mathfrak{p})$. From this, it is easy to see that the set of all $W(a)$ where $a \in \mathfrak{S}$ and $W(a) \neq \infty$, is just the ideal of the ring of ordinary integers generated by $W(\mathfrak{p})$, and since this ideal must be the whole ring of ordinary integers and $W(\mathfrak{p})>0$, then $W(\mathfrak{p})=1$ so that there exists an element $a \in \mathfrak{D}$ such that $W(a)=1$. Consequently, for each positive integer $n, W\left(a^{n}\right)=n$ so that the $\mathfrak{q}_{n}$ are all distinct and therefore, $\mathfrak{q}_{n}=\mathfrak{p}^{(n)}$. By Theorem 3, this means that for all $a \in \mathfrak{D}, W(a)$ $=V(a)$, and since $\mathfrak{D}$ is an order of $\mathfrak{D}$, one can easily show that for all $a \in \mathfrak{S}$, $W(a)=V(a)$, i.e. $W=V$.

TheOREM 9. If $\left\{W_{j}\right\}_{j \in J}$ is a set of special valuations of $\mathfrak{D}$ such that for each regular element $a \in \mathfrak{D}, W_{j}(a)=0$ for all $j \in J$ except a finite number, and if $\mathfrak{D}$ is the set of all $a \in \mathfrak{D}$ such that $W_{j}(a) \geqslant 0$ for all $j \in J$, then $\mathfrak{D}$ is a subring

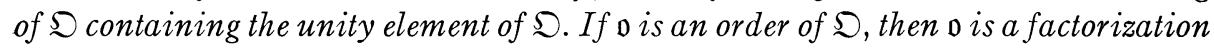
ring and if $\left\{\mathfrak{p}_{i}\right\}_{i \in I}$ is the set of relevant prime ideals of $\mathfrak{D}$, and if for each $i \in I$, $V_{i}$ is the valuation of $\mathfrak{D}$ determined by $\mathfrak{p}_{i}$, then $\left\{V_{i}\right\}_{i \in I}$ is a subset of $\left\{W_{j}\right\}_{j \in J}$.

Proof. ${ }^{2}$ Since $\mathfrak{D}$ is the intersection of the orders of $\mathfrak{D}$ determined by the $W_{j}, \mathfrak{D}$ is surely a subring of $\mathfrak{D}$ containing the unity element of $\mathfrak{D}$.

Now, let us suppose that $\mathrm{D}$ is an order of $\mathfrak{D}$. If $a \in \mathfrak{D}$ and if $b$ is a regular element of $\mathfrak{D}$ such that for all natural numbers $n, b a^{n} \in \mathfrak{D}$, this means that for all $j \in J$,

$$
W_{j}(b)+n W_{j}(a)=W_{j}\left(b a^{n}\right) \geqslant 0
$$

or $W_{j}(b) \geqslant n\left(-W_{j}(a)\right)$, and since $\infty \neq W_{j}(b) \geqslant 0,-W_{j}(a) \leqslant 0$ and $W_{j}(a) \geqslant 0$ so that $a \in \mathfrak{o}$. Thus, $\mathfrak{o}$ is fully integrally closed in $\mathfrak{D}$.

If $\mathfrak{a}, \mathfrak{b} \in \mathfrak{Q}(\mathfrak{o})$ and if $W_{j}(\mathfrak{a}) \leqslant W_{j}(\mathfrak{b})$ for all $j \in J$, then by Lemma 3 ,

$$
W_{j}\left(\mathfrak{a}^{-1} \mathfrak{b}\right)=W_{j}\left(\mathfrak{a}^{-1}\right)+W_{j}(\mathfrak{b}) \geqslant W_{j}\left(\mathfrak{a}^{-1}\right)+W_{j}(\mathfrak{a})=W_{j}\left(\mathfrak{a} \mathfrak{a}^{-1}\right) \geqslant 0
$$

${ }^{2}$ This proof is a slightly modified version of a proof given by my student M. Aubert Daigneault for the case where $\mathfrak{D}$ is an integral domain, in his Master's thesis (Université de Montréal) entitled "Les anneaux de Dedekind." 
so that $\mathfrak{a}^{-1} \mathfrak{b} \subseteq \mathfrak{D}$ and $\mathfrak{a} \leqslant \mathfrak{b}$. Therefore, $W_{j}(\mathfrak{a})=W_{j}(\mathfrak{b})$ for all $j \in J$ implies that $\mathfrak{a} \sim \mathfrak{b}$.

Furthermore, if $\mathfrak{a} \in \mathfrak{F}(\mathfrak{o})$, then $W_{j}(\mathfrak{a})=0$ for all $j \in J$ except a finite number, for there exist regular elements $a, b \in \mathfrak{D}$ such that $a \in \mathfrak{a}$ and $b \mathfrak{a} \subseteq \mathfrak{D}$ and then, for all $j \in J, W_{j}(a) \geqslant W_{j}(\mathfrak{a}) \geqslant W_{j}\left(b^{-1}\right)$ and $W_{j}(a)=W_{j}\left(b^{-1}\right)=0$ for all $j \in J$ except a finite number.

Now, if $\overline{\mathfrak{a}_{1}}>\overline{\mathfrak{a}_{2}}>\overline{\mathfrak{a}_{3}}>\ldots$ is a chain of elements of $\overline{\mathfrak{F}(\mathfrak{b})}$ where $\overline{\mathfrak{a}_{k}} \geqslant \overline{\mathfrak{b}}$ for all indices $k$, then $\mathfrak{a}_{1}{ }^{*} \subset \mathfrak{a}_{2}{ }^{*} \subset \mathfrak{a}_{3}{ }^{*} \subset \ldots$ and therefore, for all $j \in J$,

$$
W_{j}\left(\mathfrak{a}_{1}^{*}\right) \geqslant W_{j}\left(\mathfrak{a}_{2}^{*}\right) \geqslant W_{j}\left(\mathfrak{a}_{3}^{*}\right) \geqslant \ldots \geqslant 0 .
$$

Then, for each index $k, W_{j}\left(\mathfrak{a}_{k}{ }^{*}\right) \neq W_{j}\left(\mathfrak{a}_{k+1}{ }^{*}\right)$ for at least one $j \in J$ for otherwise, by what we have seen above, $\mathfrak{a}_{k}{ }^{*} \sim \mathfrak{a}_{k+1}{ }^{*}$, contradicting our hypothesis. Then, since $W_{j}\left(\mathfrak{a}_{1}{ }^{*}\right)=0$ for all $j \in J$ except a finite number, it is evident that the chain considered must be finite. Thus, $\mathfrak{D}$ is a factorization ring.

By Lemma 1 , for each $j \in J$,

$$
\mathfrak{P}_{j}=\left\{a \in \mathfrak{D} \mid W_{j}(a)>0\right\}
$$

is a regular prime ideal of $\mathfrak{D}$. If $\mathfrak{p}_{i}$ is a relevant prime ideal of $\mathfrak{D}, \mathfrak{p}_{i}$ contains a regular element $a$ which is not a unit of $\mathfrak{o}$ so that $W_{j}(a)>0$ for at least one $j \in J$. Let $J_{a}$ denote the finite set of those indices $j \in J$ for which $W_{j}(a)>0$, and for each $j \in J$, let $\mathfrak{q}_{j}$ denote the set of all $b \in \mathfrak{D}$ for which $W_{j}(b) \geqslant W_{j}(a)$. Then, $\mathfrak{q}_{j} \neq \mathrm{D}$ if and only if $j \in J_{a}$ and by Lemma 1 , for each $j \in J_{a}$, $\mathfrak{q}_{j}$ is $\mathfrak{P}_{j}$-primary. It is evident that

$$
a \in \bigcap_{j \in J a} \mathfrak{q}_{j}=\bigcap_{j \in J} \mathfrak{q}_{j} .
$$

If $b$ is in the intersection of all the $\mathfrak{q}_{j}$, for all $j \in J, W_{j}(b) \geqslant W_{j}(a)$ so that $W_{j}\left(b a^{-1}\right)=W_{j}(b)-W_{j}(a) \geqslant 0$ and therefore, $b a^{-1} \in \mathfrak{o}$ and $b \in(a)$. Therefore,

$$
\text { (a) }=\bigcap_{j \in J_{a}} \mathfrak{q}_{j}
$$

Since $\mathfrak{p}_{i} \supseteq(a), \mathfrak{p}_{i}$ contains one of the $\mathfrak{P}_{j}$ with $j \in J_{a}$, say $\mathfrak{p}_{i} \supseteq \mathfrak{P}_{j_{1}}$. Then, since $\mathfrak{P}_{j_{1}}$ is a regular prime ideal of $\mathfrak{D}$ and $\mathfrak{p}_{1}$ is a minimal regular prime ideal of $\mathfrak{o}, \mathfrak{p}_{i}=\mathfrak{P}_{j_{1}}$ and by Lemma $4, V_{i}=W_{j_{1}}$.

Theorem 10. If $V$ is a special valuation of $\mathfrak{D}$ and if $\mathrm{D}$ is the order of $\mathfrak{D}$ determined by $V$, then $V_{\mathfrak{o}}$ induces an isomorphism of $\overline{\mathcal{Q}(\mathfrak{D})}$ onto $G^{\prime \prime}$, this isomorphism mapping $\overline{\mathfrak{F}(\mathfrak{D})}$ onto $G$.

Proof. By Lemma $3, V_{\mathfrak{b}}$ is a homomorphism of $\mathfrak{l}(\mathfrak{b})$ onto $G^{\prime \prime}$. If $\mathfrak{a}, \mathfrak{b} \in \mathfrak{l}(\mathfrak{b})$,

$$
\begin{aligned}
\mathfrak{a} \leqslant \mathfrak{b} & \leftrightarrow \mathfrak{a}^{-1} \subseteq \mathfrak{b}^{-1} \\
& \leftrightarrow(a \in \mathfrak{D})(a \mathfrak{a} \subseteq \mathfrak{v} \rightarrow a \mathfrak{b} \subseteq \mathfrak{v}) \\
& \leftrightarrow(a \in \mathfrak{D})(V(a \mathfrak{a}) \geqslant 0 \rightarrow V(a \mathfrak{b}) \geqslant 0) \\
& \leftrightarrow(a \in \mathfrak{D})(V(a)+V(\mathfrak{a}) \geqslant 0 \rightarrow V(a)+V(\mathfrak{b}) \geqslant 0) \\
& \leftrightarrow V(\mathfrak{a}) \leqslant V(\mathfrak{b}) .
\end{aligned}
$$


Therefore, also $\mathfrak{a} \sim \mathfrak{b}$ if and only if $V(\mathfrak{a})=V(\mathfrak{b})$ and $V_{0}$ induces an isomorphism of $\overline{\Omega(D)}$ onto $G^{\prime \prime}$.

By Theorem 9, $\mathfrak{D}$ is a factorization ring with a single relevant prime ideal $\mathfrak{p}$ and $V$ is the valuation of $\mathfrak{D}$ determined by $\mathfrak{p}$. It is evident that for each non-negative integer $n, V\left(\mathfrak{p}^{n}\right)=n$. Since $\mathfrak{p}^{n}\left(\mathfrak{p}^{n}\right)^{-1} \subseteq \mathfrak{o}, V\left(\mathfrak{p}^{n}\left(\mathfrak{p}^{n}\right)^{-1}\right) \geqslant 0$. If we suppose that $V\left(\mathfrak{p}^{n}\left(\mathfrak{p}^{n}\right)^{-1}\right)>0$, then $\mathfrak{p}^{n}\left(\mathfrak{p}^{n}\right)^{-1} \subseteq \mathfrak{p}$ so that $\mathfrak{p}^{n}\left(\mathfrak{p}^{n}\right)^{-1} \geqslant \mathfrak{p}$ and $\mathfrak{p}^{n} \geqslant \mathfrak{p}^{n+1}$, a contradiction. Therefore,

$$
V\left(\mathfrak{p}^{n}\right)+V\left(\left(\mathfrak{p}^{n}\right)^{-1}\right)=V\left(\mathfrak{p}^{n}\left(\mathfrak{p}^{n}\right)^{-1}\right)=0
$$

so that $V\left(\left(p^{n}\right)^{-1}\right)=-n$. Therefore, $V_{\mathfrak{o}}$ maps $\mathfrak{F}(\mathfrak{p})$ onto $G$.

In Lemmas $5,6,7$ and $8, \mathfrak{D}$ is to be considered as an order of $\mathfrak{D}$ with the property that for each regular element $a \in \mathfrak{D}$ that is not a unit of $\mathfrak{D},(a)$ is the intersection of a finite number of formal powers of minimal regular prime ideals of $\mathfrak{D}$. In all of these lemmas, we may set aside the trivial case where $\mathfrak{D}=\mathfrak{D}$ so that the minimal regular prime ideals of $\mathfrak{D}$ are all proper.

Lemma 5. If $\mathfrak{p}$ is a minimal regular prime ideal of $\mathfrak{b}$, then for each positive integer $n, \mathfrak{p}^{(n)} \sim \mathfrak{p}^{n}$.

Proof. Since $\mathfrak{p}^{n} \subseteq \mathfrak{p}^{(n)},\left(\mathfrak{p}^{n}\right)^{-1} \supseteq\left(\mathfrak{p}^{(n)}\right)^{-1}$. If $a \in\left(\mathfrak{p}^{n}\right)^{-1}, a=b / c$ where $b, c \in \mathfrak{D}$ and $c$ is regular. If $c$ is a unit of $\mathfrak{D}$, then $a \in \mathfrak{D} \subseteq\left(\mathfrak{p}^{(n)}\right)^{-1}$. If $c$ is not a unit of D,

$$
(c)=\mathfrak{p}_{1}^{\left(n_{1}\right)} \cap \mathfrak{p}_{2}^{\left(n_{2}\right)} \cap \ldots \cap \mathfrak{p}_{r}^{\left(n_{r}\right)},
$$

where $\mathfrak{p}_{1}, \mathfrak{p}_{2}, \ldots, \mathfrak{p}_{r}$ are distinct minimal regular prime ideals of $\mathfrak{o}$. Since $b c^{-1} \mathfrak{p}^{n} \subseteq \mathfrak{v}, b \mathfrak{p}^{n} \subseteq(c)$. If $\mathfrak{p}$ is different from each $\mathfrak{p}_{i}$, then

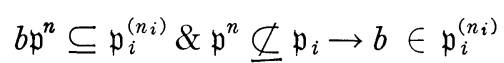

and therefore, $b \in(c), a=b / c \in \mathfrak{D} \subseteq\left(\mathfrak{p}^{(n)}\right)^{-1}$. If $\mathfrak{p}$ is equal to one of the $\mathfrak{p}_{i}$, say $\mathfrak{p}=\mathfrak{p}_{1}$, then, by the same argument as above,

$$
b \in \mathfrak{p}_{2}^{\left(n_{2}\right)} \cap \ldots \cap \mathfrak{p}_{r}^{\left(n_{r}\right)} .
$$

Then, if $d \in \mathfrak{p}^{(n)}$, there exists $g \in \mathfrak{D}$ such that $g \notin \mathfrak{p}$ and $g d \in \mathfrak{p}^{n}$ so that

$$
g(b d)=b(g d) \in(c) \subseteq \mathfrak{p}^{\left(n_{1}\right)}
$$

also

$$
g \notin \mathfrak{p} \rightarrow b d \in \mathfrak{p}^{\left(n_{1}\right)} \rightarrow b \mathfrak{p}^{(n)} \subseteq \mathfrak{p}^{\left(n_{1}\right)}
$$

and therefore,

$$
b \mathfrak{p}^{(n)} \subseteq \mathfrak{p}_{1}^{\left(n_{1}\right)} \cap \mathfrak{p}_{2}^{\left(n_{2}\right)} \cap \ldots \cap \mathfrak{p}_{r}^{\left(n_{r}\right)}=(c) .
$$

Therefore, $a \mathfrak{p}^{(n)}=b c^{-1} \mathfrak{p}^{(n)} \subseteq \mathfrak{D}$ so that $a \in\left(\mathfrak{p}^{(n)}\right)^{-1}$ and $\left(\mathfrak{p}^{n}\right)^{-1}=\left(\mathfrak{p}^{(n)}\right)^{-1}$.

Lemma 6. If $\mathfrak{p}_{1}$ and $\mathfrak{p}_{2}$ are two distinct minimal regular prime ideals of $\mathfrak{0}$ and if $k_{1}$ and $k_{2}$ are two positive integers, then

$$
\mathfrak{p}_{1}^{\left(k_{1}\right)}+\mathfrak{p}_{2}^{\left(k_{2}\right)} \sim \mathfrak{D} .
$$


Proof.

$$
\mathfrak{p}_{1}^{\left(k_{1}\right)}+\mathfrak{p}_{2}^{\left(k_{2}\right)} \subseteq \mathfrak{o} \rightarrow\left(\mathfrak{p}_{1}^{\left(k_{1}\right)}+\mathfrak{p}_{2}^{\left(k_{2}\right)}\right)^{-1} \supseteq \mathfrak{o}
$$

If

$$
a \in\left(\mathfrak{p}_{1}^{\left(k_{1}\right)}+\mathfrak{p}_{2}^{\left(k_{2}\right)}\right)^{-1},
$$

$a=b / c$ where $b, c \in \mathfrak{D}$ and $c$ is regular, and

$$
\begin{aligned}
a\left(\mathfrak{p}_{1}^{\left(k_{1}\right)}+\mathfrak{p}_{2}^{\left(k_{2}\right)}\right) \subseteq \mathfrak{v} & \rightarrow a \mathfrak{p}_{r}^{\left(k_{r}\right)} \subseteq \mathfrak{v} \\
& \rightarrow b \mathfrak{p}_{r}^{\left(k_{r}\right)} \subseteq(c) .
\end{aligned}
$$

Of course, if $c$ is a unit of $\mathfrak{D}$, then $a \in \mathfrak{D}$ and there is nothing more to prove so that we may suppose that $c$ is not a unit of $\mathfrak{D}$ and therefore,

$$
(c)=\mathfrak{p}_{i_{1}}^{\left(n_{1}\right)} \cap \mathfrak{p}_{i_{2}}^{\left(n_{2}\right)} \cap \ldots \cap \mathfrak{p}_{i_{r}}^{\left(n_{r}\right)}
$$

where

$$
\mathfrak{p}_{i_{1}}, \mathfrak{p}_{i_{2}}, \ldots, \mathfrak{p}_{i_{r}},
$$

are distinct minimal regular prime ideals of $\mathfrak{b}$. If $\mathfrak{p}_{1}$ is different from each $\mathfrak{p}_{i j}$, then

$$
b \mathfrak{p}_{1}^{\left(k_{1}\right)} \subseteq \mathfrak{p}_{i_{j}}^{\left(n_{j}\right)} \& \mathfrak{p}_{1}^{\left(k_{1}\right)} \not \subset \mathfrak{p}_{i_{j}} \rightarrow b \in \mathfrak{p}_{i_{j}}^{\left(n_{j}\right)} \quad(j \leqslant r),
$$

and therefore, $b \in(c)$ and $a=b / c \in \mathfrak{D}$. Similarly, if $\mathfrak{p}_{2}$ is different from each $\mathfrak{p}_{i j}$, one may show that $a \in \mathfrak{D}$.

Now, if

$$
\mathfrak{p}_{1}=\mathfrak{p}_{i_{1}}, \mathfrak{p}_{2}=\mathfrak{p}_{i_{2}}
$$

then

$$
b \mathfrak{p}_{1}^{\left(k_{1}\right)} \subseteq \mathfrak{p}_{i_{j}}^{\left(n_{j}\right)} \& \mathfrak{p}_{1}^{\left(k_{1}\right)} \not \subset \mathfrak{p}_{i_{j}} \rightarrow b \in \mathfrak{p}_{i_{j}}^{\left(n_{j}\right)} \quad(1<j \leqslant r)
$$

and

$$
b \mathfrak{p}_{2}^{\left(k_{2}\right)} \subseteq \mathfrak{p}_{i_{1}}^{\left(n_{1}\right)} \& \mathfrak{p}_{2}^{\left(k_{2}\right)} \not \subset \mathfrak{p}_{i_{1}} \rightarrow b \in \mathfrak{p}_{i_{1}}^{\left(n_{1}\right)},
$$

so that $b \in(c)$ and $a=b / c \in \mathfrak{D}$. Therefore

$$
\left(\mathfrak{p}_{1}^{\left(k_{1}\right)}+\mathfrak{p}_{2}^{\left(k_{2}\right)}\right)=\mathfrak{D} \text {. }
$$

Lemma 7. If $\mathfrak{p}_{1}, \mathfrak{p}_{2}, \ldots, \mathfrak{p}_{r}$ are distinct minimal regular prime ideals of $\mathfrak{D}$ and if $n_{1}, n_{2}, \ldots, n_{r}$ are positive integers, then

$$
\mathfrak{p}_{1}^{\left(n_{1}\right)} \cap \mathfrak{p}_{2}^{\left(n_{2}\right)} \cap \ldots \cap \mathfrak{p}_{r}^{\left(n_{r}\right)} \sim \mathfrak{p}_{1}^{n_{1}} \mathfrak{p}_{2}^{n_{2}} \ldots \mathfrak{p}_{r}^{n_{r}}
$$

Proof. By induction from Lemmas 5 and 6, using Propositions 3 and 4.

Lemma 8. If $\mathfrak{p}$ is a minimal regular prime ideal of $\mathfrak{b}$, then $\mathfrak{p}>\mathfrak{b}, \overline{\mathfrak{p}}$ is invertible and for each positive integer $n,\left(\mathfrak{p}^{n}\right)^{*}=\mathfrak{p}^{(n)}$.

Proof. Let $a$ be a regular element of $\mathfrak{p}$ contained in $\mathfrak{p}$. Then,

$$
(a)=\mathfrak{p}_{1}^{\left(n_{1}\right)} \cap \mathfrak{p}_{2}^{\left(n_{2}\right)} \cap \ldots \cap \mathfrak{p}_{r}^{\left(n_{r}\right)}
$$


where $\mathfrak{p}_{1}, \mathfrak{p}_{2}, \ldots, \mathfrak{p}_{r}$ are distinct minimal regular prime ideals of $\mathfrak{o}$, and $\mathfrak{p}$ must coincide with one of the $\mathfrak{p}_{i}$, say $\mathfrak{p}=\mathfrak{p}_{1}$. By Lemma 7 ,

$$
(a) \sim \mathfrak{p}_{1}^{n_{1}} \mathfrak{p}_{2}^{n_{2}} \ldots \mathfrak{p}_{r}^{n_{r}}
$$

so that

$$
p_{1}\left(p_{1}^{n_{1}-1} p_{2}^{n_{2}} \ldots p_{r}^{n_{r}} a^{-1}\right) \sim \mathfrak{D}
$$

i.e., $\bar{p}$ is invertible.

If $p \sim \mathfrak{p}$, then

$$
\mathfrak{p}_{1}^{n_{1}} \sim \mathfrak{D} \text { so that }(a) \sim \mathfrak{p}_{2}^{n_{2}} \ldots \mathfrak{p}_{r}^{n_{r}}
$$

and since $(a)=(a)^{*}$,

$$
\mathfrak{p} \supseteq(a) \supseteq \mathfrak{p}_{2}^{n_{2}} \ldots \mathfrak{p}_{\tau}^{n_{r}}
$$

so that $\mathfrak{p}=\mathfrak{p}_{1}$ contains some $\mathfrak{p}_{i}$ with $i \neq 1$, contradicting the hypothesis that the $\mathfrak{p}_{i}$ are all distinct. Therefore, $\mathfrak{p}>\mathfrak{p}$.

Since $\bar{p}$ is invertible, for each natural number $n, \overline{p^{n}}$ is invertible and by Proposition $6, \mathfrak{p}^{n}:\left(\mathfrak{p}^{n}\right)^{*} \sim \mathfrak{p}^{n}\left(\mathfrak{p}^{n}\right)^{-1} \sim \mathfrak{o}$ so that there exists $b \in \mathfrak{o}$ such that $b \notin \mathfrak{p}$ and $b\left(\mathfrak{p}^{n}\right)^{*} \subseteq \mathfrak{p}^{n} \subseteq \mathfrak{p}^{(n)}$ and therefore, $\left(\mathfrak{p}^{n}\right)^{*} \subseteq \mathfrak{p}^{(n)}$ and by Lemma 5 , $\left(\mathfrak{p}^{n}\right)^{*}=\mathfrak{p}^{(n)}$.

THEOREM 11. If $\mathrm{D}$ is an order of $\mathfrak{D}$ with the property that for each regular element $a \in \mathrm{D}$ that is not a unit of $\mathrm{D},(a)$ is the intersection of a finite number of formal powers of minimal regular prime ideals of $\mathfrak{D}$, then $\mathrm{D}$ is a factorization ring.

Proof. Let $\left\{\mathfrak{p}_{i}\right\}_{i \in I}$ be the set of all minimal regular prime ideals of $\mathfrak{D}$. For each $i \in I$, because of the properties of $\mathfrak{p}_{i}$ given by Lemma 8 , we may define a special valuation $V_{i}$ of $\mathfrak{D}$ in exactly the same way as we defined the valuation of $\mathfrak{S}$ determined by a relevant prime ideal of a factorization ring having $\mathfrak{D}$ as full ring of quotients in the preceding section. Then, if $a \in \mathfrak{o}$, it is evident that for all $i \in I, V_{i}(a) \geqslant 0$. Conversely, if $a \in \mathfrak{D}$ and if $V_{i}(a) \geqslant 0$ for all $i \in I$, then $a=b / c$ where $b, c \in \mathbb{D}$ and $c$ is regular, and if $c$ is not a unit of o, then

$$
(c)=\mathfrak{p}_{i_{1}}^{\left(n_{1}\right)} \cap \mathfrak{p}_{i_{2}}^{\left(n_{2}\right)} \cap \ldots \cap \mathfrak{p}_{i_{r}}^{\left(n_{r}\right)}
$$

where $i_{1}, i_{2}, \ldots, i_{r}$ are distinct elements of $I$, and one can easily verify that for $j \leqslant r, V_{i j}(c)=n_{j}$, while for $i \in I, i \neq i_{j}, V_{i}(c)=0$. Then,

$$
V_{i_{j}}(a)=V_{i_{j}}(b)-V_{i_{j}}(c) \geqslant 0 \rightarrow V_{i_{j}}(b) \geqslant V_{i_{j}}(c) \rightarrow b \in \mathfrak{p}_{i_{j}}^{\left(n_{j}\right)} \quad(j \leqslant r)
$$

and therefore, $b \in(c)$ and $a=b / c \in \mathfrak{D}$. Therefore, by Theorem $9, \mathfrak{D}$ is a factorization ring.

6. Generalized Dedekind rings. We will say that a factorization ring $\mathfrak{D}$ is a generalized Dedekind ring, if for $\mathfrak{a}, \mathfrak{b} \in \mathfrak{F}(\mathfrak{b}), \mathfrak{a} \sim \mathfrak{b}$ implies that $\mathfrak{a}=\mathfrak{b}$. This means that $\mathfrak{F}(\mathfrak{D})$ is isomorphic to $\overline{\mathfrak{F}(\mathfrak{D})}$ so that every proper regular ideal 
of $\mathfrak{o}$ may be expressed in a unique way as the product of a finite number of relevant prime ideals of $\mathfrak{D}$.

If $\mathfrak{D}$ is an order of $\mathfrak{D}$, then $\mathfrak{D}$ is a generalized Dedekind ring if and only if 0 satisfies one of the following sets of conditions:

1. $\mathfrak{F}(\mathfrak{D})$ is a group.

2. For any two regular ideals $\mathfrak{a}$ and $\mathfrak{b}$ of $\mathfrak{b}, \mathfrak{a} \subseteq \mathfrak{b}$ implies that there exists an ideal $\mathfrak{c}$ of $\mathfrak{D}$ (it must be regular) such that $\mathfrak{a}=\mathfrak{b} c$.

3. (i) $\mathrm{D}$ is a factorization ring,

(ii) the relevant prime ideals of $\mathfrak{v}$ are the only proper regular prime ideals of $\boldsymbol{p}$.

4. (i) $\mathbb{D}$ is fully integrally closed in $\mathfrak{D}$,

(ii) if $\mathfrak{a}$ is any regular ideal of $\mathfrak{b}$, then the descending chain condition is valid for the ideals of $\mathfrak{o} / \mathfrak{a}$.

We will not prove the equivalence of these sets of conditions, the proofs being entirely similar to those given in the case of integral domains.

For simplicity, we will use the term "Dedekind ring" instead of "generalized Dedekind ring" and "Dedekind domain" instead of "ordinary Dedekind ring."

THEOREM 12. If $\mathfrak{D}$ is a Dedekind ring with $\mathfrak{D}$ as full ring of quotients and if $V$ is a special valuation of $\mathfrak{S}$ with the property that $V(a) \geqslant 0$ for all $a \in \mathrm{o}$, then $V$ is determined by a relevant prime ideal of $\mathrm{D}$.

Proof. By Lemma $1, \mathfrak{p}=\{a \in \mathfrak{o} \mid V(a)>0\}$ is a proper regular prime ideal of $\mathfrak{p}$, and since $\mathfrak{D}$ is a Dedekind ring, $\mathfrak{p}$ is a relevant prime ideal of $\mathfrak{o}$ so that by Lemma 4, $V$ is determined by $p$.

Theorem 13. If $V$ is a special valuation of $\mathfrak{D}$, if $\mathfrak{o}$ is the order of $\mathfrak{O}$ determined by $V$ and if $\mathfrak{D}$ satisfies either one of the following two conditions:

1. there is only a finite number of maximal prime ideals of $(0)$ in $\mathfrak{O}$,

2. $\mathfrak{O}$ is "einartig" (4, p. 22),

then $\mathrm{o}$ is a Dedekind ring.

Proof. Let $\mathfrak{p}$ denote the unique relevant prime ideal of $\mathfrak{b}$.

1. Let us assume that there is only a finite number of maximal prime ideals $\mathfrak{P}_{1}, \mathfrak{P}_{2}, \ldots, \mathfrak{P}_{n}$ of $(0)$ in $\mathfrak{O}$ (this condition is satisfied when the ascending chain condition holds for the ideals of $\mathfrak{D}$ and a fortiori, when it holds for the ideals of $\mathfrak{p}$ ). Let $\mathfrak{a}$ be a proper ideal of $\mathfrak{p}$. If $a \in \mathfrak{a}$ and $a \notin \mathfrak{p}$, then $V(a)=0$ and $a$ is not a regular element of $\mathfrak{b}$, for if $a$ were regular, $V\left(a^{-1}\right)=-V(a)=0$ so that $a^{-1} \in \mathfrak{D}$ and $\mathfrak{a}$ would not be a proper ideal of $\mathfrak{D}$. Then,

$$
a \in\left(\mathfrak{P}_{1} \cup \mathfrak{B}_{2} \cup \ldots \cup \mathfrak{P}_{n}\right) \cap \mathfrak{p}=\left(\mathfrak{B}_{1} \cap \mathfrak{p}\right) \cup\left(\mathfrak{P}_{2} \cap \mathfrak{p}\right) \cup \ldots \cup\left(\mathfrak{P}_{n} \cap \mathfrak{o}\right)
$$

and therefore,

$$
\mathfrak{a} \subseteq \mathfrak{p} \cup\left(\mathfrak{P}_{1} \cap \mathfrak{p}\right) \cup\left(\mathfrak{P}_{2} \cap \mathfrak{o}\right) \cup \ldots \cup\left(\mathfrak{B}_{n} \cap \mathfrak{o}\right)
$$

Since the ideals $\mathfrak{p}, \mathfrak{P}_{1} \cap \mathfrak{p}, \mathfrak{P}_{2} \cap \mathfrak{b}, \ldots, \mathfrak{P}_{\mathfrak{n}} \cap \mathfrak{p}$ are prime ideals of $\mathfrak{o}, \mathfrak{a}$ 
must be contained in one of them. But if $\mathfrak{a} \subseteq\left(\mathfrak{B}_{i} \cap \mathfrak{o}\right)$, $\mathfrak{a}$ is not regular. Therefore, $\mathfrak{p}$ is the only proper regular prime ideal of $\mathfrak{D}$.

2. Let us assume that $\mathfrak{D}$ is "einartig" (this condition is certainly satisfied when the descending chain condition is valid for the ideals of $\mathfrak{D}$ ). By Lemma 1 , the associate $\mathfrak{p}^{\prime}$ of $\mathfrak{p}$ is a proper prime ideal of $\mathfrak{D}$ so that it must be a maximal proper ideal of $\mathfrak{D}$ and $\mathfrak{D} / \mathfrak{p}^{\prime}$ is a field. Since $\mathfrak{p}^{\prime}$ is the kernel of $V$, we may speak of the projection $V^{\prime}$ of $V$ by the natural homomorphism of $\mathfrak{S}$ onto $\mathfrak{D} / \mathfrak{p}^{\prime}$. Then, $\mathfrak{o} / \mathfrak{p}^{\prime}$ is the order of $\mathfrak{D} / \mathfrak{p}^{\prime}$ determined by $V^{\prime}$ (rank 1 , discrete valuation of the field $\mathfrak{D} / \mathfrak{p}^{\prime}$ ) so that $\mathfrak{p} / \mathfrak{p}^{\prime}$ is the only non-trivial prime ideal of $\mathfrak{o} / \mathfrak{p}^{\prime}$ and $\mathfrak{p}$ is the only proper regular prime ideal of $\mathfrak{D}$.

For the remainder of this section, we will assume that $\mathfrak{o}$ is a Dedekind ring and that the ascending chain condition is valid for the ideals of $\mathbf{0}$. We will denote the set of all regular elements of $\mathfrak{o}$ by $S,\left\{\mathfrak{p}_{i}\right\}_{i \in I}$ will be the set of relevant prime ideals of $\mathfrak{D}$ and for each $i \in I, V_{i}$ will denote the valuation of $\mathfrak{D}=\mathfrak{o}_{S}$ determined by $\mathfrak{p}_{i}$. If $\mathfrak{a}$ is an ideal of $\mathfrak{o}, I(\mathfrak{a})$ will denote the set of all $i \in I$ for which $0<V_{i}(\mathfrak{a})<\infty$ and $I^{\prime}(\mathfrak{a})$ will denote the set of all $i \in I$ for which $V_{i}(\mathfrak{a})=\infty$. If $i \in I$, since

$$
\mathfrak{p}_{i}^{\prime}=\bigcap_{n=1}^{\infty} \mathfrak{p}_{i}^{(n)}=\bigcap_{n=1}^{\infty} \mathfrak{p}_{i}^{n}
$$

we will set $\mathfrak{p}_{i}{ }^{\prime}=\mathfrak{p}_{i}{ }^{(\infty)}=\mathfrak{p}_{i}{ }^{\infty}$.

THEOREM 14. If $\mathfrak{a}$ is an ideal of $\mathfrak{b}$, then $I(\mathfrak{a})$ is finite, for each $i \in I(\mathfrak{a}), \mathfrak{a}_{\boldsymbol{s}}$ and $\mathfrak{p}_{i}$ are without proper common divisor and

$$
\mathfrak{a}=\mathfrak{a}_{S} \cdot \prod_{i \in I(\mathfrak{a})} \mathfrak{p}_{i}^{m_{i}}
$$

where $m_{i}=V_{i}(\mathfrak{a})$. We will call this representation of $\mathfrak{a}$ the standard decomposition of $\mathfrak{a}$.

Proof. Let $\mathfrak{a}=\mathfrak{q}_{1} \cap \mathfrak{q}_{2} \cap \ldots \cap \mathfrak{q}_{n}$ be a normal decomposition of $\mathfrak{a}$ into primary ideals. The radicals of some of these primary ideals may be relevant prime ideals of $\mathfrak{D}$. Let us say that the radicals of $\mathfrak{q}_{1}, \mathfrak{q}_{2}, \ldots, \mathfrak{q}_{r}(0 \leqslant r \leqslant n)$ are the relevant prime ideals

$$
\mathfrak{p}_{i_{1}}, \mathfrak{p}_{i_{2}}, \ldots, \mathfrak{p}_{i_{r}}
$$

respectively $\left(i_{j} \in I\right)$, while the radicals of $\mathfrak{q}_{r+1}, \ldots, \mathfrak{q}_{n}$ are not relevant prime ideals of $\mathfrak{D}$. Since the relevant prime ideals of $\mathfrak{D}$ are the only proper regular prime ideals of $\mathfrak{D}$,

$$
\mathfrak{a}_{S}=\mathfrak{q}_{r+1} \cap \ldots \cap \mathfrak{q}_{n} .
$$

By Theorem 6 , for each $k \leqslant r, \mathfrak{q}_{k}$ is a formal power, say

$$
\mathfrak{q}_{k}=\mathfrak{p}_{i_{k}}^{(n k)}
$$

and since $\mathfrak{o}$ is a Dedekind ring,

$$
\mathfrak{q}_{1} \cap \mathfrak{q}_{2} \cap \ldots \cap \mathfrak{q}_{r}=\mathfrak{p}_{i_{1}}^{\left(n_{1}\right)} \cap \mathfrak{p}_{i_{2}}^{\left(n_{2}\right)} \cap \ldots \cap \mathfrak{p}_{i_{r}}^{\left(n_{r}\right)}=\mathfrak{p}_{i_{1}}^{n_{1} \mathfrak{p}_{i_{2}}^{n_{2}} \ldots \mathfrak{p}_{i_{r}}^{n_{r}}}
$$


Now, if $h>r$, then the radical of $\mathfrak{q}_{h}$ is not contained in any of the ideals

$$
\mathfrak{p}_{i_{1}}, \mathfrak{p}_{i_{2}}, \ldots, \mathfrak{p}_{i_{r}},
$$

for if

$$
\operatorname{rad} \mathfrak{q}_{h} \subseteq \mathfrak{p}_{i_{k}} \quad(1 \leqslant k \leqslant r),
$$

since $\operatorname{rad} \mathfrak{q}_{h}$ is a prime ideal of $\mathfrak{b}$, but not a relevant prime ideal of $\mathfrak{o}$, by Theorem 4,

$$
\operatorname{rad} \mathfrak{q}_{h} \subseteq \mathfrak{p}_{i_{k}}^{\prime}, \mathfrak{q}_{k}=\mathfrak{p}_{i_{k}}^{n_{k}} \supseteq \mathfrak{p}_{i_{k}}^{\prime} \supseteq \mathfrak{q}_{h},
$$

contradicting the hypothesis that the given decomposition of $\mathfrak{a}$ into primary ideals is normal. From this, we deduce first of all, since the p's are maximal proper ideals of $\mathfrak{b}$, that $\mathfrak{a}_{S}$ and each $\mathfrak{p}_{i_{k}}$ are without proper common divisors, so that

$$
\mathfrak{a}=\mathfrak{p}_{i_{1}}^{n_{1}} \mathfrak{p}_{i_{2}}^{n_{2}} \ldots \mathfrak{p}_{i_{r}}^{n_{r}} \cap \mathfrak{a}_{S}=p_{i_{1}}^{n_{1}} p_{i_{2}}^{n_{2}} \ldots p_{i_{r}}^{n_{r}} \mathfrak{a}_{S}
$$

Secondly, for each $k \leqslant r, \mathfrak{p}_{i k}$ is a minimal prime ideal of $\mathfrak{a}$ and

$$
\begin{gathered}
\mathfrak{a}_{\mathfrak{p}_{i k}}=\mathfrak{p}_{i k}^{n_{k}} \rightarrow \mathfrak{a} \subseteq \mathfrak{p}_{i k}^{n k} \text { and } \mathfrak{a} \not \subset \mathfrak{p}_{i k}^{n_{k}+1} \\
\rightarrow n_{k}=V_{i_{k}}(\mathfrak{a})=m_{k} \text { and }\left\{i_{1}, i_{2}, \ldots, i_{r}\right\} \subseteq I(\mathfrak{a})
\end{gathered}
$$

Now, let us assume that $i \in I$ and that $i \notin\left\{i_{1}, i_{2}, \ldots, i_{r}\right\}$. First of all, if $\mathfrak{a} \not \subset \mathfrak{p}_{i}$, then $V_{i}(\mathfrak{a})=0$ and $i \notin I(\mathfrak{a})$. Secondly, if $\mathfrak{a} \subseteq \mathfrak{p}_{i}$, since $\mathfrak{p}_{i}$ does not belong to $\mathfrak{a}$, all prime ideals belonging to $\mathfrak{a}$ and contained in $\mathfrak{p}_{i}$ must be contained in $\mathfrak{p}_{i}^{\prime}$ (Theorem 4). Since $\mathfrak{p}_{i}^{\prime}$ is a minimal prime ideal of $\mathfrak{p}, \mathfrak{p}_{i}^{\prime}$ is the only prime ideal belonging to $\mathfrak{a}$ and contained in $\mathfrak{p}_{i}$ so that $V_{i}(\mathfrak{a})=\infty$ and $i \notin I(\mathfrak{a})$.

Theorem 14 implies that an ideal $\mathfrak{a}$ of $\mathfrak{b}$ is completely determined by its isolated component $\mathfrak{a}_{S}$ and by its values $V_{i}(\mathfrak{a})$, for all $i \in I(\mathfrak{a})$.

Let us notice that if $\mathfrak{a}$ and $\mathfrak{b}$ are ideals of $\mathfrak{b}$, then by Lemma 3 ,

$$
\begin{aligned}
& V_{i}(\mathfrak{a} \mathfrak{b})=V_{i}(\mathfrak{a})+V_{i}(\mathfrak{b}), \\
& V_{i}(\mathfrak{a}+\mathfrak{b})=\min \left\{V_{i}(\mathfrak{a}), V_{i}(\mathfrak{b})\right\},
\end{aligned}
$$

and by one of the remarks made after Theorem 7 , if $V_{i}(\mathfrak{a})=m_{i}$ and $V_{i}(\mathfrak{b})=n_{i}$, then

$$
(\mathfrak{a} \cap \mathfrak{b})_{\mathfrak{p}_{i}}=\mathfrak{a}_{\mathfrak{p}_{i}} \cap \mathfrak{b}_{\mathfrak{p}_{i}}=\mathfrak{p}_{i}^{m_{i}} \cap \mathfrak{p}_{i}^{n_{i}}=\mathfrak{p}_{i}^{\max \left\{m_{i}, n_{\mathfrak{i}}\right\}},
$$

so that $V_{i}(\mathfrak{a} \cap \mathfrak{b})=\max \left\{V_{i}(\mathfrak{a}), V_{i}(\mathfrak{b})\right\}$. These rules are useful for finding $I(\mathfrak{a} \mathfrak{b}), I(\mathfrak{a}+\mathfrak{b})$ and $I(\mathfrak{a} \cap \mathfrak{b})$ when the sets $\left\{V_{i}(\mathfrak{a})\right\}_{i \in I}$ and $\left\{V_{i}(\mathfrak{b})\right\}_{i \in I}$ are given.

The standard decomposition of an ideal $\mathfrak{a}$ of $\mathfrak{b}$ is not the only representation of $\mathfrak{a}$ as the product of $\mathfrak{a}_{S}$ with a finite number of positive powers of relevant prime ideals of $\mathfrak{p}$. For example, it $\mathfrak{p}$ is a relevant prime ideal of $\mathfrak{p}$ and if $\boldsymbol{p}^{\prime}$ is the associate of $\mathfrak{p}$, then $\mathfrak{p}^{\prime}=\left(\mathfrak{p}^{\prime}\right)_{s}$ is the standard decomposition of $\mathfrak{p}^{\prime}$. But since

$$
\mathfrak{p}^{\prime}=\bigcap_{n=1}^{\infty} \mathfrak{p}^{n}
$$


by Krull's Intersection Theorem, $\mathfrak{p}^{\prime}=(0)_{s^{\prime}}$, where $S^{\prime}$ is the set of all elements of $\mathfrak{D}$ that are of the form $1-a$, where $a \in \mathfrak{p}$. Then, if $b \in \mathfrak{p}^{\prime}$, there exists $a \in \mathfrak{p}$ such that $(1-a) b=0$ so that $b=a b \in \mathfrak{p} \mathfrak{p}^{\prime}$ and $\mathfrak{p}^{\prime}=\mathfrak{p} \mathfrak{p}^{\prime}=\mathfrak{p}\left(\mathfrak{p}^{\prime}\right)_{S}$.

TheOREM 15. If $\mathfrak{a}$ is an ideal of $\mathfrak{D}$ and if

$$
\mathfrak{a}=\mathfrak{a}_{S} \prod_{i \in I(\mathfrak{a})} \mathfrak{p}_{i}^{m_{i}}
$$

is the standard decomposition of $\mathfrak{a}$, then any representation of $\mathfrak{a}$ as the product of $\mathfrak{a}_{S}$ with a finite number of positive powers of relevant prime ideals of $\mathfrak{b}$ is of the form

$$
\mathfrak{a}=\mathfrak{a}_{S} \prod_{i \in I(\mathfrak{a})} \mathfrak{p}_{i}^{m_{i}} \prod_{i \in J} \mathfrak{p}_{i}^{n_{i}},
$$

where $J$ is a finite subset of $I^{\prime}(\mathfrak{a})$.

Proof. Let us suppose that

$$
\mathfrak{a}_{S} \prod_{i \in I(\mathfrak{a})} \mathfrak{p}_{i}^{m_{i}}=\mathfrak{a}_{S} \prod_{i \in K} \mathfrak{p}_{i}^{n_{i}}
$$

where $K$ is a finite subset of $I$ and $n_{i}>0$ for all $i \in K$. If $j \in I(\mathfrak{a})$, by Theorem $14, \mathfrak{p}_{j}$ and $\mathfrak{a}_{S}$ are without proper common divisor so that $\mathfrak{a}_{S} \not \subset \mathfrak{p}_{j}$, and since

$$
\mathfrak{p}_{j} \supseteq \mathfrak{a}_{S} \prod_{i \in K} \mathfrak{p}_{i}^{n_{i}}
$$

$\mathfrak{p}_{j}$ must contain, and therefore be equal to, some $\mathfrak{p}_{i}$ for $i \in K$. Since $\mathfrak{p}_{j}$ is invertible, we may cancel $p_{j}$ from both sides of (1). It is then evident that we may repeat this argument until all the relevant prime ideals appearing on the left-hand side of (1) have been cancelled so that $I(\mathfrak{a})$ is a subset of $K$, for each $i \in I(\mathfrak{a}), m_{i} \leqslant n_{i}$ and if we set $J=K-I(\mathfrak{a})$,

$$
\mathfrak{a}_{S}=\mathfrak{a}_{S} \prod_{i \in I(\mathfrak{a})} \mathfrak{p}_{i}^{n_{i}-m_{i}} \prod_{i \in J} \mathfrak{p}_{i}^{n_{i}} .
$$

From this equation, since for each $i \in I(\mathfrak{a}), \mathfrak{p}_{i} \nsupseteq \mathfrak{a}_{S}$, it is evident that $m_{i}=n_{i}$ so that

$$
\mathfrak{a}_{S}=\mathfrak{a}_{S} \prod_{i \in J} \mathfrak{p}_{i}^{n_{i}}
$$

But if $i \in J, \mathfrak{p}_{i} \supseteq \mathfrak{a}$ and therefore, $V_{i}(\mathfrak{a})>0$. But then, since $i \notin I(\mathfrak{a})$, $V_{i}(\mathfrak{a})=\infty$ and $i \in I^{\prime}(\mathfrak{a})$.

7. A special case. In this section, we will assume that the descending chain condition is valid for the ideals of $\mathfrak{D}$ and our object will be to determine all orders of $\mathfrak{D}$ that are Noetherian Dedekind rings.

If $\mathfrak{N}_{1}, \mathfrak{N}_{2}, \ldots, \mathfrak{N}_{p}$ are the proper prime ideals of $\mathfrak{D}$, then

$$
(0)=\bigcap_{h=1}^{p} \mathfrak{Q}_{h} \quad \text { (direct intersection), }
$$

where each $\mathfrak{Q}_{h}$ is $\mathfrak{N}_{h}$-primary, and 


$$
\mathfrak{D}=\sum_{k=1}^{p} \mathfrak{D}_{k}
$$

where

$$
\mathfrak{D}_{k}=\bigcap_{\substack{h=1 \\ h \neq k}}^{p} \mathfrak{\varrho}_{h} \quad \text { and } \quad \mathfrak{Q}_{h}=\sum_{\substack{k=1 \\ k \neq h}}^{p} \mathfrak{D}_{k} .
$$

Also, $\mathfrak{D}_{k} \cong \mathfrak{D} / \mathfrak{Q}_{k}$ so that $\mathfrak{D}_{k}$ is completely primary, i.e., every non-regular element of $\mathfrak{D}_{k}$ is nilpotent.

If for each $k=1,2, \ldots, p, \mathfrak{D}_{k}$ is an order of $\mathfrak{D}_{k}$, then it is easy to show that

$$
\mathfrak{D}=\sum_{k=1}^{p} \mathfrak{o}_{k}
$$

is an order of $\mathfrak{D}$ and that $\mathfrak{D}$ is fully integrally closed in $\mathfrak{D}$ if and only if each $\mathfrak{D}_{k}$ is fully integrally closed in $\mathfrak{D}_{k}$. If $\mathfrak{D}$ is any order of $\mathfrak{D}$ and if $\Phi_{k}$ is the projection function of $\mathfrak{D}$ onto $\mathfrak{S}_{k}$, then $\Phi_{k}(\mathfrak{D})$ is an order of $\mathfrak{D}_{k}$ and if

$$
\mathfrak{D}=\sum_{k=1}^{p} \Phi_{k}(\mathfrak{D})
$$

then we will say that $\mathbf{D}$ is decomposable.

Lemma 9. If $\mathfrak{D}$ is an order of $\mathfrak{D}$, then $\mathfrak{D}$ is decomposable if and only if $h \neq k$ implies that $\left(\mathfrak{\bigcap}_{h} \cap \mathfrak{D}\right)+\left(\mathfrak{\complement}_{k} \cap \mathfrak{D}\right)=\mathfrak{D},(h, k=1,2, \ldots, p)$.

Proof. Suppose that

$$
\mathfrak{D}=\sum_{k=1}^{p} \mathfrak{o}_{k}
$$

where each $\mathfrak{o}_{k}$ is an order of $\mathfrak{S}_{k}$. Then evidently,

$$
\mathfrak{Q}_{h} \cap \mathfrak{v}=\sum_{\substack{k=1 \\ k \neq h}}^{p} \mathfrak{b}_{k},
$$

so that $h \neq k$ implies that $\left(\mathfrak{Q}_{h} \cap \mathfrak{D}\right)+\left(\mathfrak{Q}_{k} \cap \mathfrak{D}\right)=\mathfrak{D}$.

Conversely, if $h \neq k$ implies that $\left(\mathfrak{Q}_{h} \cap \mathfrak{D}\right)+\left(\mathfrak{\Omega}_{k} \cap \mathfrak{D}\right)=\mathfrak{D}$, and if we set $\mathfrak{q}_{h}=\mathfrak{\Omega}_{h} \cap \mathfrak{o}$, then, in $\mathfrak{D}$,

$$
(0)=\bigcap_{h=1}^{p} \mathfrak{q}_{h}
$$

(direct intersection)

so that

$$
\mathfrak{o}=\sum_{k=1}^{p} \mathfrak{o}_{k}
$$

where

$$
\mathbf{v}_{k}=\bigcap_{\substack{h=1 \\ h \neq k}}^{p} \mathfrak{q}_{h} \subseteq \mathfrak{D}_{k}
$$


Corollary. If $\mathfrak{o}$ and $\mathfrak{D}^{\prime}$ are orders of $\mathfrak{D}$, if $\mathfrak{o} \subseteq \mathfrak{o}^{\prime}$ and if $\mathfrak{o}$ is decomposable, then $\mathfrak{D}^{\prime}$ is decomposable.

Proof. $h \neq k$ implies that

$$
1 \in \mathfrak{v}=\left(\mathfrak{Q}_{h} \cap \mathfrak{o}\right)+\left(\mathfrak{\Omega}_{k} \cap \mathfrak{o}\right) \subseteq\left(\mathfrak{Q}_{h} \cap \mathfrak{o}^{\prime}\right)+\left(\mathfrak{Q}_{k} \cap \mathfrak{o}^{\prime}\right)
$$

Let us suppose that for $k \leqslant q(0 \leqslant q \leqslant p), \mathfrak{Q}_{k}=\mathfrak{N}_{k}$, i.e. $\mathfrak{O}_{k} \cong \mathfrak{D} / \mathfrak{Q}_{k}$ is a field, while for $k>q, \mathfrak{D}_{k} \subset \mathfrak{N}_{k}$. We consider a commutative ring with unity element in which every regular element is invertible as a Dedekind ring with no relevant prime ideals.

THEOREM 16. If for each $k=1,2, \ldots, p, \mathfrak{D}_{k}$ is an order of $\mathfrak{D}_{k}$, if for each $k \leqslant q, \mathfrak{D}_{k}$ is a Dedekind domain and if for $k>q, \mathfrak{D}_{k}=\mathfrak{D}_{k}$, then

$$
\mathfrak{v}=\sum_{k=1}^{p} \mathfrak{o}_{k}
$$

is a Noetherian Dedekind ring, and every order of $\mathfrak{D}$ that is a Noetherian Dedekind ring may be obtained in this way. If $\mathfrak{D}_{k} \subset \mathfrak{D}_{k}$ for each $k \leqslant s(0 \leqslant s \leqslant q)$ and $\mathbf{0}_{k}=\mathfrak{D}_{k}$ for $k>s$, and if, for each $k \leqslant s, \mathfrak{n}_{k}$ is the kernel of $\Phi_{k}$ in $\mathfrak{D}$, then $\left\{\mathfrak{n}_{k}\right\}_{k \leqslant s}$ is the set of associates of relevant prime ideals of $\mathfrak{D}$ and any ideal of $\mathfrak{D}$ that contains $\mathfrak{n}_{k}$ properly $(k \leqslant s)$ must be regular.

Proof. Let us assume first of all that for each $k \leqslant p, \mathrm{D}_{k}$ is an order of $\mathfrak{D}_{k}$ obeying the conditions of the theorem. Then,

$$
\mathfrak{o}=\sum_{k=1}^{p} \mathfrak{o}_{k}
$$

is an order of $\mathfrak{D}$, and since each $\mathfrak{D}_{k}$ is fully integrally closed in $\mathfrak{D}_{k}$, $\mathfrak{D}$ is fully integrally closed in $\mathfrak{D}$. Furthermore, it is evident that $\mathfrak{D}$ is a Noetherian. Let $\mathfrak{a}$ be a regular ideal of $\mathfrak{b}, \mathfrak{a}=\mathfrak{a}_{1}+\mathfrak{a}_{2}+\ldots+\mathfrak{a}_{p}$, where $\mathfrak{a}_{k}$ is an ideal of $\mathfrak{o}_{k}$. Since $\mathfrak{a}$ contains a regular element $a$ of $\mathfrak{D}$ and since $a=a_{1}+a_{2}+\ldots+a_{p}$, where $a_{k}$ is a regular element of $\mathfrak{b}_{k}$, each $\mathfrak{a}_{k}$ is a regular ideal of $\mathfrak{b}_{k}$. Then,

$$
\frac{\mathfrak{D}}{\mathfrak{a}}=\sum_{k=1}^{p} \frac{\mathfrak{a}+\mathfrak{o}_{k}}{\mathfrak{a}} \cong \sum_{k=1}^{p} \frac{\mathfrak{o}_{k}}{\mathfrak{a} \cap \mathfrak{o}_{k}}=\sum_{k=1}^{p} \frac{\mathfrak{p}_{k}}{\mathfrak{a}_{k}}
$$

(direct sums)

and from the definition of the $\mathfrak{b}_{k}$, the descending chain condition is valid for the ideals of $\mathfrak{b}_{k} / \mathfrak{a}_{k}$, so that it is also valid for the ideals of $\mathfrak{o} / \mathfrak{a}$. Therefore, $\mathfrak{v}$ is a Dedekind ring.

Conversely, let us assume that $\mathfrak{D}$ is an order of $\mathfrak{D}$ and that $\mathfrak{D}$ is a Noetherian Dedekind ring. For each $k \leqslant p$, set $\mathfrak{n}_{k}=\mathfrak{N}_{k} \cap \mathfrak{o}$ and $\mathfrak{q}_{k}=\mathfrak{Q}_{k} \cap \mathfrak{o}$. The ideals $\mathfrak{n}_{k}$ are the only non-regular prime ideals of $\mathfrak{o}, h \neq k$ implies that $\mathfrak{n}_{h} \not \subset \mathfrak{n}_{k}$ and each $\mathfrak{q}_{k}$ is $\mathfrak{n}_{k}$-primary.

To prove that $\mathfrak{o}$ is decomposable, by Lemma 9 , all we have to show is that $h \neq k$ implies that $\mathfrak{q}_{h}+\mathfrak{q}_{k}=\mathfrak{v}$ or equivalently, that $\mathfrak{n}_{h}+\mathfrak{n}_{k}=\mathfrak{v}$. If $h \neq k$ and if $\mathfrak{n}$ is a prime ideal of $\mathfrak{D}$ containing $\mathfrak{n}_{h}+\mathfrak{n}_{k}$, then evidently, $\mathfrak{n}$ cannot be 
contained in any $\mathfrak{n}_{j}$, and furthermore, $\mathfrak{n}$ cannot be contained in any relevant prime ideal $\mathfrak{p}$ of $\mathfrak{v}$, for then, by Theorem $4, \mathfrak{n}_{h}$ and $\mathfrak{n}_{k}$ would both be contained in the associate $\mathfrak{p}^{\prime}$ and $\mathfrak{p}$ and would therefore be equal to $\mathfrak{p}^{\prime}$, a contradiction. Therefore, $\mathfrak{n}=\mathfrak{v}$ and $\mathfrak{n}_{h}+\mathfrak{n}_{k}=\mathfrak{v}$.

The associates of relevant prime ideals of $\mathfrak{o}$ must be amongst the $\mathfrak{n}_{k}$. If $\mathfrak{n}_{k}$ is the associate of a relevant prime ideal $\mathfrak{p}$ of $\mathfrak{b}$, then $\mathfrak{n t}_{k}$ is the only $\mathfrak{n}_{k}$-primary ideal of $\mathfrak{p}$ (see remarks following Theorem 7 ) so that

$$
\mathfrak{o}_{k}=\Phi_{k}(\mathfrak{D}) \cong \mathfrak{o} / \mathfrak{n}_{k}
$$

is an integral domain and not a field since it contains the non-trivial ideal $\Phi_{k}(\mathfrak{p})$ and therefore, $k \leqslant s$. Furthermore, if $\mathfrak{a}$ is an ideal of $\mathfrak{o}$ that contains $\mathfrak{n}_{k}$ properly, then $\mathfrak{a}$ is regular, for if $\mathfrak{a}$ were not regular, then $\mathfrak{D a}$ would be a proper ideal of $\mathfrak{S}$ and would therefore be contained in some $\mathfrak{R}_{j}$ and then

$$
\mathfrak{n}_{k} \subset \mathfrak{a} \subseteq ऽ \mathfrak{a} \cap \mathfrak{o} \subseteq \mathfrak{n}_{j},
$$

a contradiction. Then, a may be expressed as a product of relevant prime ideals of $\mathfrak{0}$ having $\mathfrak{n}_{k}$ as associate (standard decomposition of $\mathfrak{a}$ ) so that $\Phi_{k}(\mathfrak{a})$ may be expressed as a product of prime ideals of $\mathfrak{0}_{k}$ and therefore, $\mathfrak{0}_{k}$ is a Dedekind domain.

If $\mathfrak{n}_{k}$ is not the associate of a relevant prime ideal of $\mathfrak{o}$, then $\mathfrak{n}_{k}$ is a maximal proper ideal of $\mathfrak{D}$ so that $\mathfrak{o}_{k} \cong \mathfrak{v} / \mathfrak{q}_{k}$ is a completely primary ring and therefore, $\mathbf{0}_{k}=\mathfrak{S}_{k}$ so that $k>s$.

In the following corollaries, $\mathfrak{D}$ is a Noetherian Dedekind ring having $\mathfrak{D}$ as full ring of quotients and we adopt the notation developed in Theorem 16.

Corollary 1. If for $k \leqslant s$,

$$
\left\{p_{i}\right\}_{i \in Y_{k}}
$$

is the set of relevant prime ideals of $\mathbf{0}$ that have $\mathfrak{n}_{k}$ as associate, then

$$
\left\{\Phi_{k}\left(\mathfrak{p}_{i}\right)\right\}_{i \in I_{k}}
$$

is the set of relevant prime ideals of $\mathfrak{p}_{k}$ and for each $i_{\epsilon} I_{k}, \mathfrak{p}_{i}=\Phi_{k}{ }^{-1} \Phi_{k}\left(\mathfrak{p}_{i}\right)$.

Corollary 2. If for each $i_{\in} I_{k}, V_{i}$ is the valuation of $\mathfrak{D}$ determined by $\mathfrak{p}_{i}$ and $V_{i}^{\prime}$ is the valuation of $\mathfrak{O}_{k}$ determined by $\Phi_{k}\left(\mathfrak{p}_{i}\right)$, then $V_{i}^{\prime}$ is the projection of $V_{i} b y \Phi_{k}$.

COROLlary 3. If $\mathfrak{o}^{\prime}$ is an order of $\mathfrak{D}$ and if $\mathfrak{o} \subseteq \mathfrak{v}^{\prime}$, then $\mathfrak{o}^{\prime}$ is also a Noetherian Dedekind ring.

Proof. By the Corollary of Lemma 9, $\mathfrak{D}^{\prime}$ is also decomposable, and since for each $k, \Phi_{k}(\mathfrak{o}) \subseteq \Phi_{k}\left(\mathfrak{o}^{\prime}\right)$, for each $k>s, \Phi_{k}\left(\mathfrak{o}^{\prime}\right)=\mathfrak{D}_{k}$ and by a theorem of MacLane and Schilling (6, Lemma 37), for $k \leqslant s, \Phi_{k}\left(\mathbf{0}^{\prime}\right)$ is a Dedekind domain, so that by Theorem $16, \mathfrak{b}^{\prime}$ is a Noetherian Dedekind ring. It is evident that this Corollary is a generalization of the above mentioned Theorem of MacLane and Schilling. 


\section{REFERENCES}

1. G. Birkhoff, Lattice Theory, Amer. Math. Soc. Colloquium Publications, 25.

2. L. Fuchs, The generalization of the valuation theory, Duke Math. J., 18 (1951), 19-26.

3. W. Krull, Über die Zerlegung der Hauptideale in all-gemeinen Ringen, Math. Ann., 105 (1931), 1-14.

4. - Idealtheorie (1935; Chelsea, 1948).

5. D. G. Northcott, Ideal Theory (Cambridge, 1953).

6. O. F. G. Schilling, The Theory of Valuations, Math. Surveys of the Amer. Math. Soc., IV (1950).

7. B. L. van der Waerden, Moderne Algebra (1937; 2nd ed. New York, 1943).

\section{Université de Montréal}

